\title{
Exponential asymptotics and Stokes lines in a partial differential equation
}

\author{
By S. Jonathan Chapman and David B. Mortimer \\ Mathematical Institute, 24-29 St Giles, Oxford OX1 3LB.
}

A singularly perturbed linear partial differential equation motivated by the geometrical model for crystal growth is considered. A steepest descent analysis of the Fourier transform solution identifies asymptotic contributions from saddle points, end points and poles, and the Stokes lines across which these may be switched on and off. These results are then derived directly from the equation by optimally truncating the naïve perturbation expansion and smoothing the Stokes discontinuities. The analysis reveals two new types of Stokes switching: a higher-order Stokes line which is a Stokes line in the approximation of the late terms of the asymptotic series, and which switches on or off Stokes lines themselves; and a second-generation Stokes line, in which a subdominant exponential switched on at a primary Stokes line is itself responsible for switching on another smaller exponential. The 'new' Stokes lines discussed by Berk et al. (1982) are second-generation Stokes lines, while the 'vanishing' Stokes lines discussed by Aoki et al. (1998) are switched off by a higher-order Stokes line.

Keywords: Exponential asymptotics, Stokes lines, Stokes smoothing, Borel plane, optimal truncation

\section{Introduction}

The geometrical model for the steady growth of a single finger of a dendritic crystal written down by Brower et al. (1983) and analysed by Kruskal \& Segur (1991) is

$$
\begin{aligned}
\epsilon^{2} \phi^{\prime \prime \prime}+\phi^{\prime} & =\cos \phi, \\
\phi & \rightarrow \pm \frac{\pi}{2} \quad \text { as } s \rightarrow \pm \infty,
\end{aligned}
$$

where $s$ is arclength along the (two-dimensional) solid-liquid interface, $\phi$ is the angle that the normal to this interface makes with the $x$-axis (the direction of propagation of the finger), and ${ }^{\prime} \equiv \mathrm{d} / \mathrm{d} s$. As shown in Kruskal \& Segur (1991), a naïve asymptotic expansion

$$
\phi=\sum_{n=0}^{\infty} \epsilon^{2 n} \phi_{n}
$$

can be made to satisfy the equation and boundary conditions at each order. However, there is in fact no solution to (1.1)-(1.2), as was proved rigorously by Amick \& McLeod (1990). The series (1.3) diverges for any fixed values of $\epsilon>0$ and $s$. 
Optimally truncating this series at its least term reveals a Stokes line in the complex $s$-plane joining the points $s= \pm i \pi / 2$, which are the complex singularities of the leading-order approximation

$$
\phi_{0}=-\pi / 2+2 \tan ^{-1} \mathrm{e}^{s} .
$$

Across the Stokes line an exponentially small term "beyond all orders" is switched on as shown by Chapman et al. (1998). Although this term is exponentially small at the origin where it is turned on, it grows exponentially as $s \rightarrow \infty$ and its presence means that the boundary conditions (1.2) cannot be satisfied.

A natural question to ask now is, if we consider a time dependent problem which has (1.1) as the steady state, what will the large-time behaviour be? There is no steady finger solution, therefore, this cannot provide the large time behaviour. Since the small exponential is oscillatory it is tempting to imagine that its presence is the origin of side branching in dendritic fingers.

The "correct" time-dependent generalisation of (1.1) is

$$
v_{n}=\kappa+\epsilon^{2} \kappa_{s s}
$$

where $\kappa$ is the curvature and $v_{n}$ is the normal velocity of the solid-liquid interface Brower et al. (1983); solutions of (1.1) are travelling waves solutions of (1.5). However, we can ask the same questions about large-time behaviour for any timedependent problem for which (1.1) gives the steady state. The simplest such equation is

$$
\phi_{t}-\phi_{s}=\epsilon^{2} \phi_{s s s}-\cos \phi .
$$

The beyond all orders analysis of equations such as (1.6) is an order of magnitude harder than that of (1.1) and has yet to be attempted in the literature. To gain some insight and to illustrate the sort of issues which need to be addressed, we consider here a linearised version of (1.6) which has the advantage that we can solve using the Fourier transform to give the solution in the form of an integral. This exact solution will provide a check on our direct asymptotic techniques, so that we have more confidence in the latter when we move on to consider nonlinear equations, for which such an integral representation of the solution is usually lacking.

The specific linear problem we will consider is

$$
\begin{aligned}
\phi_{t}-\phi_{s} & =\epsilon^{2} \phi_{s s s}-\frac{1}{1+s^{2}}, \\
\phi & \rightarrow \pm \frac{\pi}{2} \quad \text { as } s \rightarrow \pm \infty .
\end{aligned}
$$

The nonlinearity has been replaced by an inhomogeneous term which introduces complex singularities at $s= \pm i$ into the solution corresponding to those at $s=$ $\pm i \pi / 2$ in the crystal growth problem. The inhomogeneous term has been chosen so that the leading order steady solution is

$$
\phi_{0}=\tan ^{-1} s,
$$

which is simpler than (1.4) but of the same general form. The steady-state problem

$$
\begin{aligned}
\phi_{s}+\epsilon^{2} \phi_{s s s} & =\frac{1}{1+s^{2}}, \\
\phi & \rightarrow \pm \frac{\pi}{2} \quad \text { as } s \rightarrow \pm \infty
\end{aligned}
$$


exhibits the same features as the crystal-growth problem in that a naïve expansion

$$
\phi=\sum_{n=0}^{\infty} \epsilon^{2 n} \phi_{n}
$$

can be made to satisfy the equation and boundary conditions at every order. There is, however, a Stokes line between the singularities $s= \pm i$ across which a subdominant exponential is switched on. In this case the exponential is oscillating but not growing or decaying at infinity, though this still fails to satisfy the boundary conditions (1.11) and thus rules out a steady finger solution. The initial condition is not so important for our analysis, but we choose to use the leading-order steady state

$$
\phi(s, 0)=\tan ^{-1} s,
$$

so that we are starting close to a finger solution and seeing how it will evolve.

In Section 2 we solve (1.7)-(1.8), (1.13) exactly using the Fourier transform and asymptotically evaluate the resulting integrals as $\epsilon \rightarrow 0$ using steepest descents. We determine the Stokes lines in real and complex $(s, t)$-space and the form of the solution in each region.

In Section 3 we analyse the equation asymptotically by truncating the asymptotic series (1.12) and examining the behaviour of the remainder. We show that the exponentials are smoothly switched on across the Stokes lines in exactly the manner discovered by Berry (1989) for linear o.d.e.s. Here in order to calculate these exponentials we follow the framework of matched asymptotic expansions set out by Daalhuis et al. (1995).

The crucial step in using optimal truncation in order to examine the remainder term is to find the late terms, that is, to determine $\phi^{(n)}$ in the limit $n \rightarrow \infty$. For this particular problem we are able to determine each term in the series exactly and then approximate the series in the large $n$ limit. In doing this we encounter a phenomenon which we shall call a higher-order Stokes line. Across this line contributions to the approximation of the late terms are switched on and off. Thus a higher-order Stokes line switches on or off Stokes lines themselves. The higher-order Stokes line is associated with what we shall call second-generation Stokes switching; a subdominant exponential switched on across a Stokes line is a divergent asymptotic series which itself switches on further terms beyond all orders. This is origin of the 'new' Stokes lines discussed by Berk et al. (1982) and the 'vanishing' Stokes lines discussed by Aoki et al. (1998).

We aim to use our techniques on nonlinear problems where an exact representation of the terms $\phi^{(n)}$ will not in general be available. With this end in mind, in Section 5 we try to obtain the late terms using a WKBJ type ansatz of the form factorial/power, as has been done by Chapman et al. (1998) for nonlinear ordinary differential equations. We will find that determining the "phase" of the approximation is possible (but not straightforward, since the boundary data may be given on a characteristic of the resulting eikonal equation), but that determining the premultiplying amplitude involves solving an inner problem analogous to the inner diffraction problems that arise in the Geometrical Theory of Diffraction (see Keller $(1958,1962))$. 
Finally, we note that in a companion paper, Howls et al. (2005) consider the same differential equation (1.7) using hyperasymptotics and Borel transforms; there the higher-order Stokes line manifests itself in the location of branch points in the Borel plane.

\section{Asymptotic analysis of the exact solution}

\section{(a) Exact solution by Fourier transform}

Consider the equation (1.10) with boundary condition (1.11) and initial condition (1.13), with $0<\epsilon \ll 1$. Defining the Fourier transform as

$$
\hat{\phi}=\int_{-\infty}^{\infty} \phi \mathrm{e}^{i k s} \mathrm{~d} s
$$

taking the Fourier transform of (1.10)-(1.11), (1.13) gives

$$
\hat{\phi}_{t}+i k \hat{\phi}=\epsilon^{2} i k^{3} \hat{\phi}-\pi \mathrm{e}^{-|k|}, \quad \hat{\phi}(0)=\frac{i \pi}{k} \mathrm{e}^{-|k|} .
$$

Hence

$$
\hat{\phi}=\frac{i \pi \mathrm{e}^{-|k|}}{k\left(1-\epsilon^{2} k^{2}\right)}\left(1-\epsilon^{2} k^{2} \mathrm{e}^{-i k\left(1-\epsilon^{2} k^{2}\right) t}\right)
$$

The inversion formula now gives

$$
\phi=I_{s}+I_{t},
$$

with

$$
\begin{aligned}
I_{s} & =\int_{-\infty}^{\infty} \frac{i \mathrm{e}^{(-|p|-i p s) / \epsilon}}{2 p\left(1-p^{2}\right)} \mathrm{d} p \\
I_{t} & =\int_{-\infty}^{\infty} \frac{-i p}{2\left(1-p^{2}\right)} \exp \left(\left(-|p|-i p\left(1-p^{2}\right) t-i p s\right) / \epsilon\right) \mathrm{d} p
\end{aligned}
$$

where, in anticipation of the approximation by steepest descents as $\epsilon \rightarrow 0$ to follow, we have put $p=k \epsilon$. For the integrals along the real axis to exist we require $-1 \leq$ $\operatorname{Im}(s) \leq 1$. Note that although the singularities of $\hat{\phi}$ at $p= \pm 1$ are removeable, so that $\hat{\phi}$ does not have any poles, when we split the integral into $I_{s}$ and $I_{t}$ there are poles in each integrand (with equal and opposite residues). We choose to indent the contours of integration clockwise around the poles at $p=-1$ and $p=1$ in both integrals, so that the pole contributions cancel initially (this will be true as long as we indent the same way in both integrals). Note that the first integral $I_{s}$ is a steady-state solution of equation (1.10), but we shall see that it does not satisfy the boundary conditions (1.11).

\section{(b) Approximation of the exact solution}

(i) Non-time dependent integrals

We first consider the steady-state solution $I_{s}$. By partial fractions we find

$$
I_{s}=\int_{0}^{\infty} \sin \left(\frac{p s}{\epsilon}\right) \frac{\mathrm{e}^{-p / \epsilon}}{p} \mathrm{~d} p+I_{s 1}+I_{s 2},
$$


where

$$
I_{s 1}=\int_{0}^{\infty} \frac{i p \mathrm{e}^{-p(1+i s) / \epsilon}}{2\left(1-p^{2}\right)} \mathrm{d} p, \quad I_{s 2}=-\int_{0}^{\infty} \frac{i p \mathrm{e}^{-p(1-i s) / \epsilon}}{2\left(1-p^{2}\right)} \mathrm{d} p,
$$

where we have removed the modulus sign by splitting the range of integration and putting $p \rightarrow-p$ in the integral over $(-\infty, 0)$. The contour is indented clockwise around $p=1$ in $I_{s 1}$ and anti-clockwise around $p=1$ in $I_{s 2}$.

The first integral in (2.6) can be evaluated exactly by noting that

$$
\frac{\partial}{\partial s}\left(\int_{0}^{\infty} \sin \left(\frac{p s}{\epsilon}\right) \frac{\mathrm{e}^{-p / \epsilon}}{p} \mathrm{~d} p\right)=\int_{0}^{\infty} \cos \left(\frac{p s}{\epsilon}\right) \mathrm{e}^{-p / \epsilon} \frac{\mathrm{d} p}{\epsilon}=\frac{1}{1+s^{2}} .
$$

Thus

$$
\int_{0}^{\infty} \sin \left(\frac{p s}{\epsilon}\right) \frac{\mathrm{e}^{-p / \epsilon}}{p} \mathrm{~d} p=\tan ^{-1} s
$$

Noting that there are no saddle points in $I_{s_{1}}$, we apply the method of steepest descents as $\epsilon \rightarrow 0$ to the integral $I_{s_{1}}$. We get no pole contribution when the indented contour can be deformed into the steepest descent contour $\arg (p(1+i s))=0$ without passing through $p=1$. This is the case for $\operatorname{Re}(s)<0$. When $\operatorname{Re}(s)=0$ the steepest descent contour arg $p=0$ passes through the pole, while for $\operatorname{Re}(s)>0$ the steepest descent contour must be indented to include a clockwise circuit around the pole (Figure 1). Thus, as the real part of $s$ increases through zero we pick up an extra
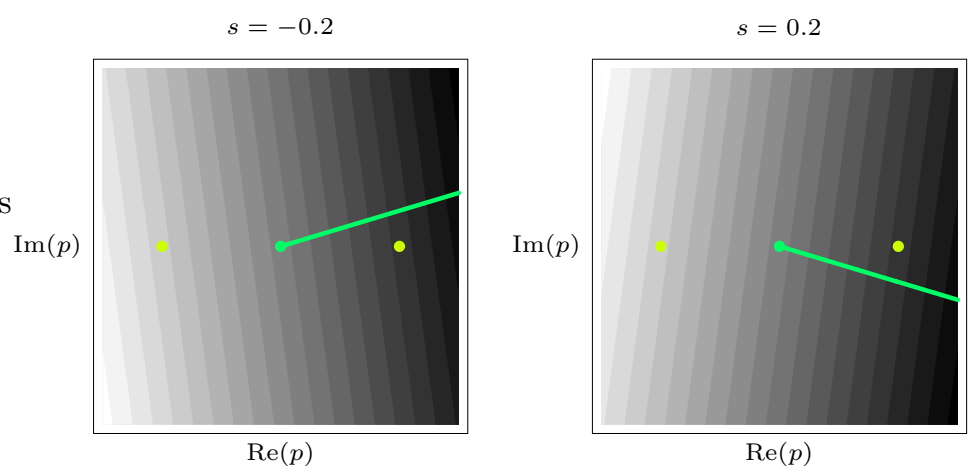

Figure 1. Steepest descent paths for $I_{s 1}$.

pole contribution, and

$$
\phi_{p 1}=-2 \pi i \operatorname{Res}\left(\frac{i p \mathrm{e}^{-p(1+i s) / \epsilon}}{2\left(1-p^{2}\right)}, p=1\right)=-\frac{\pi}{2} \mathrm{e}^{-(1+i s) / \epsilon},
$$

is switched on. A similar analysis can be performed upon $I_{s 2}$, with the contour crossing the pole from below to above as $s$ increases, so that as the real part of $s$ increases through zero we pick up a contribution from integrating anti-clockwise around the pole at $p=1$, and

$$
\phi_{p 2}=2 \pi i \operatorname{Res}\left(-\frac{i p \mathrm{e}^{-p(1-i s) / \epsilon}}{2\left(1-p^{2}\right)}, p=1\right)=-\frac{\pi}{2} \mathrm{e}^{-(1-i s) / \epsilon}
$$


is switched on. Combining these, we see that as we cross $\operatorname{Re}(s)=0$ a contribution

$$
\phi_{p}=\phi_{p 1}+\phi_{p 2}=-\pi \mathrm{e}^{-1 / \epsilon} \cos \left(\frac{s}{\epsilon}\right)
$$

is switched on. In addition the end point contributions from $I_{s 1}$ and $I_{s 2}$ are

$$
\phi_{s e}=\phi_{s e 1}+\phi_{s e 2} \sim \frac{i \epsilon^{2}}{2(1+i s)^{2}}-\frac{i \epsilon^{2}}{2(1-i s)^{2}},
$$

and are present everywhere.

(ii) Time dependent integrals

We now consider the integral $I_{t}$. We again remove the modulus sign in the integral by splitting the range of integration and putting $k \rightarrow-k$ in the integral over $(-\infty, 0)$, to give

$$
I_{t}=I_{t 1}+I_{t 2}
$$

where

$$
I_{t 1}=\int_{0}^{\infty} \frac{-i p \mathrm{e}^{-f(p) / \epsilon}}{2\left(1-p^{2}\right)} \mathrm{d} p, \quad I_{t 2}=\int_{0}^{\infty} \frac{i p \mathrm{e}^{-\bar{f}(p) / \epsilon}}{2\left(1-p^{2}\right)} \mathrm{d} p
$$

where

$$
f(p)=i p s+p+i p\left(1-p^{2}\right) t
$$

a bar denotes complex conjugation, and the contour is indented clockwise around $p=1$ in $I_{t 1}$ and anticlockwise around $p=1$ in $I_{t 2}$. We apply the method of steepest descent to $I_{t_{1}}$ as $\epsilon \rightarrow 0$. In addition to the endpoint at $p=0$ and the poles at $p= \pm 1$, there are now two saddle points to consider, at

$$
p=p_{s^{ \pm}}= \pm\left(\frac{s+t-i}{3 t}\right)^{1 / 2}
$$

We wish to know which of these five possible contributions is present in the expansion of the integral at each point in the $(s, t)$-plane. There can only be a change in the set of contributions across Stokes lines, where the contour through one of these points passes through another. Since there can therefore only be a Stokes line where two contributions have the same phase, we first consider the equal phase lines associated with each pair of contributions.

There are three possible Stokes switchings, as listed below.

1. The contribution from a pole may be switched on or off by the contour through the end point

When the steepest descent contour through the end point crosses a pole the contribution from the pole will be switched on or off. On the line where this happens the phases of the end point and pole contributions are equal, i.e. $\operatorname{Im}(f( \pm 1))=$ $\operatorname{Im}(f(0))$. Thus $\pm \operatorname{Im}(i s+1)=0$, that is $\operatorname{Re}(s)=0$. The height of the pole must also be less than that of the endpoint, i.e. $\operatorname{Re}(f( \pm 1))>\operatorname{Re}(f(0))$. 
2. The contribution from a pole may be switched on or off by a contour through a saddle point When the steepest descent contour through a saddle point crosses a pole the contribution from the pole will be switched on or off. On the line where this happens the phases of the saddle point and pole contributions are equal, i.e. $\operatorname{Im}(f( \pm 1))=\operatorname{Im}\left(f\left(p_{s^{ \pm}}\right)\right)$. Thus

$$
\mp \frac{2}{\sqrt{27 t}} \operatorname{Im}\left(i(s+t-i)^{3 / 2}\right)=\mp \operatorname{Im}(i s+1) .
$$

The height of the pole must also be less than that of the saddle point, i.e. $\operatorname{Re}(f( \pm 1))>$ $\operatorname{Re}\left(f\left(p_{s^{ \pm}}\right)\right)$.

3. The contribution from a saddle point may be switched on or off by the contour through the end point When the steepest descent contour through the end point passes through a saddle point the contribution from the saddle point will be switched on or off. On the line where this happens the phases of the end point and saddle point contributions are equal, i.e. $\operatorname{Im}\left(f\left(p_{s^{ \pm}}\right)\right)=\operatorname{Im}(f(0))$. Thus

$$
\mp \frac{2}{\sqrt{27 t}} \operatorname{Im}\left(i(s+t-i)^{3 / 2}\right)=0,
$$

which implies

$$
\operatorname{Im}(s)=1 \pm \sqrt{3}(t+\operatorname{Re}(s)) \quad \text { or } \quad \operatorname{Im}(s)=1 .
$$

The height of the saddle point must also be less than that of the endpoint, i.e. $\operatorname{Re}\left(f\left( \pm p_{s^{ \pm}}\right)\right)>\operatorname{Re}(f(0))$.

We now need to find out if any of these Stokes switchings are active. We are interested in what happens in real space so we first plot all possible Stokes lines in the real $(s, t)$-plane; these are shown in Figure 2. The long dashed line corresponds to 1 , the short dashed line corresponds to 2 and the solid black line to 3 .

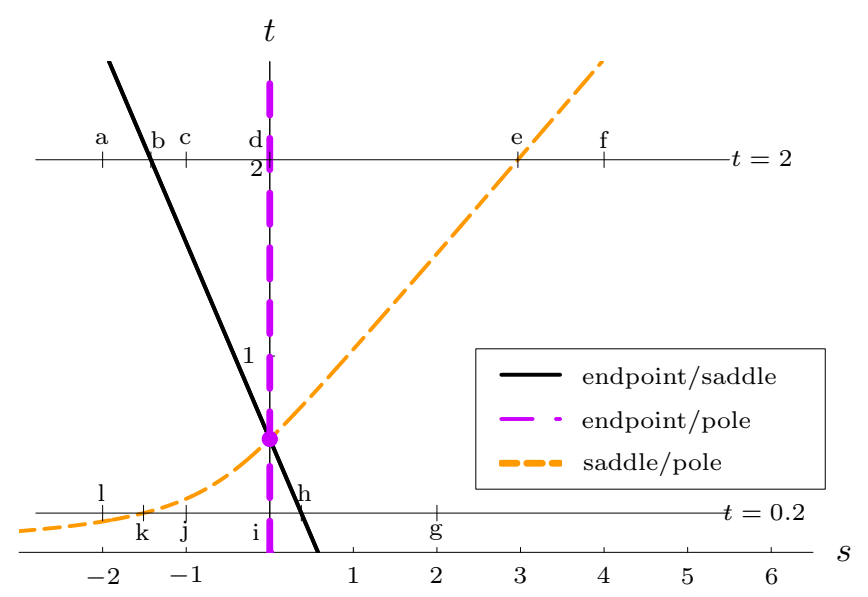

Figure 2. Possible Stokes lines in real space. Labels a, b, etc. refer to Figures 3 and 4.

We see from this diagram that there is a crucial time $t=1 / \sqrt{3}$ where the Stokes lines intersect. We consider the steepest descent paths before and after this time. 


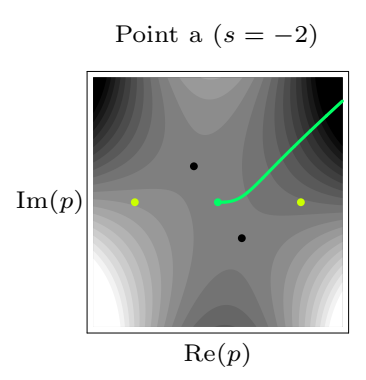

Point d $(s=0)$

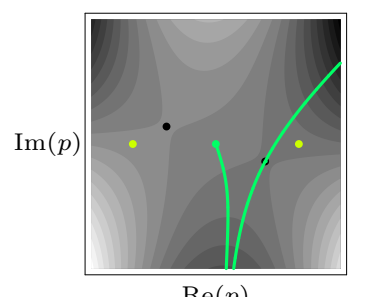

$\operatorname{Re}(p)$

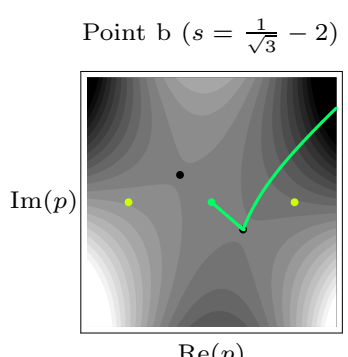

$\operatorname{Re}(p)$

Point e $(s=2.97 .$.

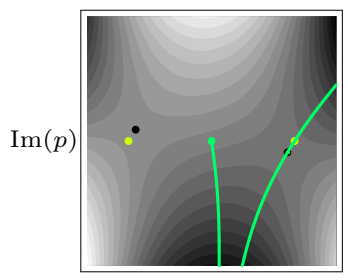

$\operatorname{Re}(p)$
Point c $(s=-1)$

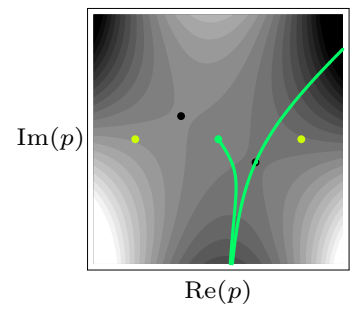

Point f $(s=4)$

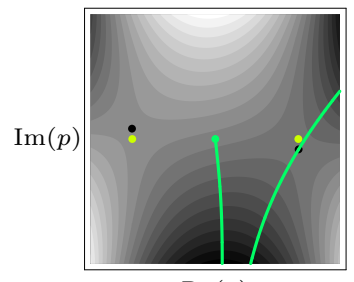

$\operatorname{Re}(p)$

Figure 3. Steepest descent paths at $t=2$. Labels a,b,..,g refer to points on Figure 2.

We first consider the paths as $\operatorname{Re}(s)$ varies for fixed $t>1 / \sqrt{3}$. For $t=2$ a selection of these paths are shown in Figure 3. The first thing to note is that the endpoint will always contribute to the approximation of the integral. To leadingorder this contribution is

$$
\phi_{t e 1} \sim \frac{i \epsilon^{2}}{2(s+t-i)^{2}}
$$

Note that this contribution is singular at $s=-t+i$, where the saddle point coalesces with the endpoint.

We see from Figure 3 that for $s<1 / \sqrt{3}-t$ (see point a) the saddle does not contribute. As $s$ crosses this line (corresponding to the solid black line in Figure 2) the saddle $p_{s^{+}}$is picked up by the steepest descent curve (see point b), and the saddle point contribution is switched on. To leading-order, this contribution is

$$
\phi_{s 1} \sim \frac{\mathrm{e}^{3 i \pi / 4} \sqrt{\pi \epsilon}(3 t)^{1 / 4}(s+t-i)^{1 / 4}}{2(2 t-s+i)} \exp \left(-\frac{i}{\epsilon} \sqrt{\frac{4(s+t-i)^{3}}{27 t}}\right) .
$$

Note that this contribution has a singularity at $s=2 t+i$ corresponding to the saddle point coalescing with a pole. Also the exponent of $\phi_{s 1}$ has a branch point at $s=-t+i$ corresponding to the saddle point coalescescing with the end point.

We see that no new contribution is switched on or off across $s=0$ (the long dashed line in Figure 2, see point d) so this line is inactive. The endpoint and the pole have the same phase at this point, but they are not "adjacent". Finally a contribution from the pole at $p=1$ is switched on as the contour through the saddle point crosses it, (corresponding to the short dashed line in Figure 2, see 
point e). To leading-order, the pole contribution is

$$
-2 \pi i \operatorname{Res}\left(\frac{-i p \mathrm{e}^{-\left(i p s+p+i p\left(1-p^{2}\right) t\right) / \epsilon}}{2\left(1-p^{2}\right)}, p=1\right)=\frac{\pi}{2} \mathrm{e}^{-(1+i s) / \epsilon}=-\phi_{p 1} .
$$

Note that this pole contribution is equal and opposite to that from the timeindependent integral (2.10).

We now consider the steepest descent paths as $s$ varies in $\mathbb{R}$ for fixed $t<1 / \sqrt{3}$. A selection of these for $t=0.2$ are shown in Figure 4 . As $s$ crosses the line $s=1 / \sqrt{3}-t$

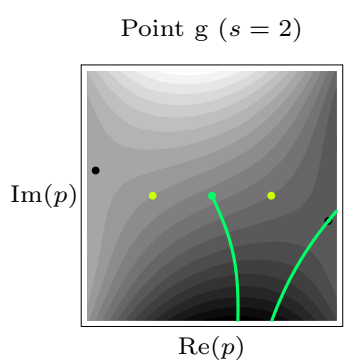

Point j $(s=-1)$

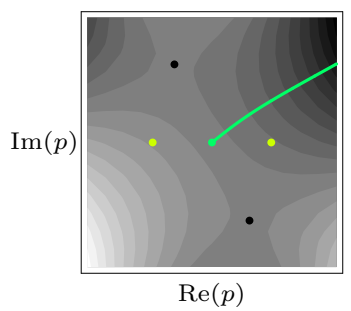

Point h $\left(s=\frac{1}{\sqrt{3}}-0.2\right)$

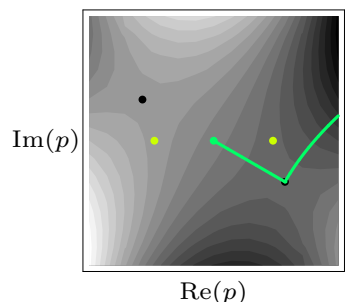

Point $\mathrm{k}(s=-1.51 .$.

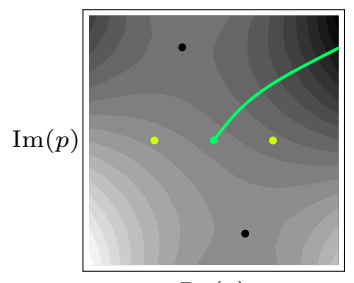

$\operatorname{Re}(p)$
Point i $(s=0)$

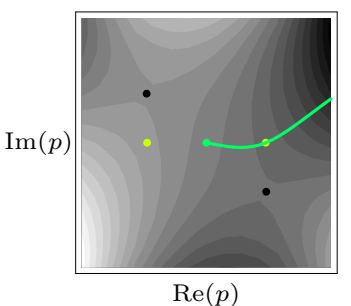

Point $\mathrm{l}(s=-2)$

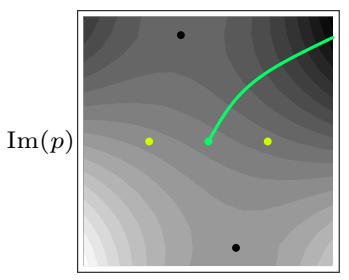

$\operatorname{Re}(p)$

Figure 4. Steepest descent paths at $t=0.2 \mathrm{~g}, \mathrm{~h}, \ldots, 1$ refer to points on figure 2 .

(corresponding to the solid black line in Figure 2, see point $\mathrm{h}$ ) the steepest descent path through the endpoint crosses the saddle point, the saddle $p_{s^{+}}$is lost from the steepest descent curve, and the saddle point contribution is switched off.

As $s$ decreases further, it crosses the point $s=0$ (corresponding to the long dashed line in Figure 2, see point i). Here the steepest descent contour through the endpoint crosses the pole, and the pole contribution is switched off.

Note that as $s$ crosses the short dashed line in Figure 2 (point $\mathrm{k}$ ) no further switching occurs. Across this line the steepest descent path though the saddle crosses the pole. However, the integration contour does not go through the saddle point; there is no saddle point contribution to switch on the pole.

Thus, our final Stokes line picture in real $(s, t)$-space is as shown in Figure 5 , where we have set $\phi_{t e}=\phi_{t e 1}+\phi_{t e 2}$ and $\phi_{s}=\phi_{s 1}+\phi_{s 2}$.

Consider now the integral $I_{t 2}$. We find that we get exactly the same picture of Stokes lines in real space as in Figure 5.

To leading-order, the end point contribution is

$$
\phi_{t e 2} \sim-\frac{i \epsilon^{2}}{2(s+t+i)^{2}} ;
$$




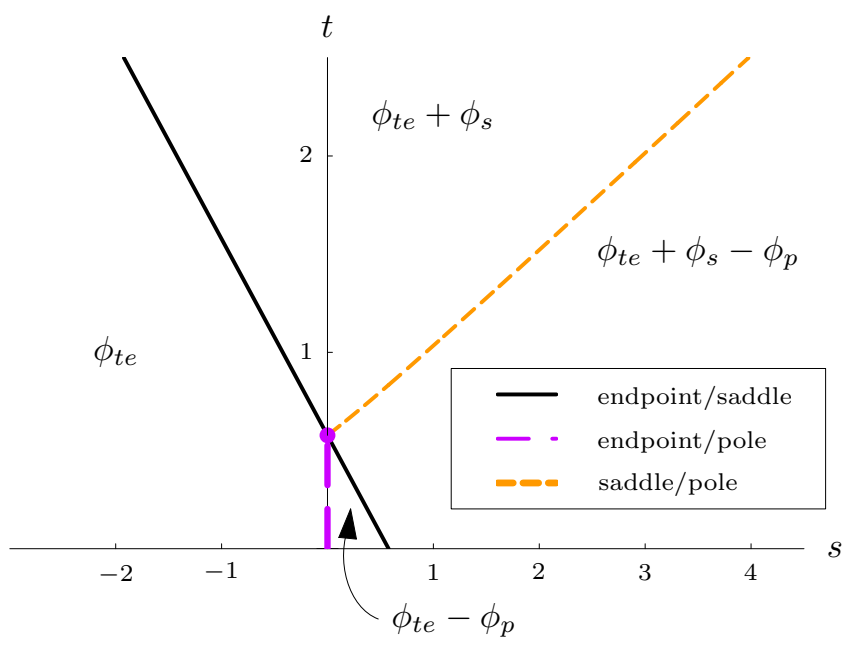

Figure 5. Active Stokes lines in real space $I_{t 1}$.

the saddle point contribution is

$$
\phi_{s 2} \sim \frac{\mathrm{e}^{-3 i \pi / 4} \sqrt{\pi \epsilon}(3 t)^{1 / 4}(s+t+i)^{1 / 4}}{2(2 t-s-i)} \exp \left(\frac{i}{\epsilon} \sqrt{\frac{4(s+t+i)^{3}}{27 t}}\right)
$$

and the pole contribution is

$$
-\phi_{p 2} \sim \frac{\pi}{2} \mathrm{e}^{-(1-i s) / \epsilon}
$$

Having approximated $I_{s}$ and $I_{t}$, we can now combine the results to create a picture of the complete Stokes structure as shown in Figure 6 , in which $\phi_{e}=\phi_{t e}+\phi_{s e}+$ $\tan ^{-1} s$.

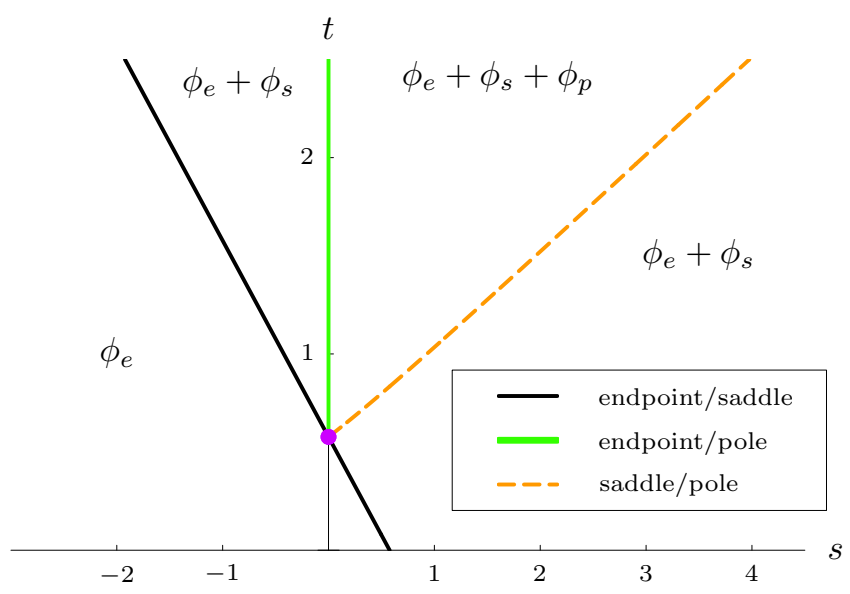

Figure 6. Stokes lines in real space.

There are two aspects of the Stokes line structure that require further comment, even though neither is central to the analysis in the present paper. The first is the 
fact that the solid black Stokes line, across which the endpoint switches on the saddle point, intersects the $t=0$ axis. However, we know that the solution at $t=0$ is simply the initial condition $\tan ^{-1} s$, which has a truncating series in $\epsilon$ (of one term), and thus cannot exhibit Stokes phenomenon.

The resolution of this apparent paradox can be seen by examining the position of the saddle point in the integral $I_{t 1}$, which we found to be

$$
p_{s}=\left(\frac{s+t-i}{3 t}\right)^{1 / 2} \text {. }
$$

We see that this saddle comes in from infinity at $t=0+$. If we repeat our steepest descent analysis for $t=\mathcal{O}(\epsilon)$ we will find endpoint and pole contributions as before, but no saddle point in the integral. Thus there is a boundary layer at $t=0$ in which the saddle point and Stokes line appear.

The saddle point contribution switched on across the Stokes line,

$$
\begin{array}{r}
\phi_{s} \sim-\frac{\sqrt{\pi \epsilon}(3 t)^{1 / 4}}{2}\left(\frac{\mathrm{e}^{3 i \pi / 4}}{(s-i)^{3 / 4}} \exp \left(-\frac{i}{\epsilon} \sqrt{\frac{4(s-i)^{3}}{27 t}}\right)+\right. \\
\left.\frac{\mathrm{e}^{-3 i \pi / 4}}{(s+i)^{3 / 4}} \exp \left(\frac{i}{\epsilon} \sqrt{\frac{4(s+i)^{3}}{27 t}}\right)\right),
\end{array}
$$

as $t \rightarrow 0$. Thus this term tends to zero exponentially quickly as $t \rightarrow 0$.

This phenomenon of a saddle suddenly appearing from infinity at $t=0+$ will be generic in problems of this type. It occurs because the third derivative in the equation gives a term $t k^{3}$ in the exponent of the Fourier transform, while the initial data gives an exponent linear in $k$ for large $k$. Thus there are two more saddle points when $t>0$ than when $t=0$.

The second aspect on which we need to comment is the existence of Stokes lines parallel to the real $s$-axis (the second solution in (2.19)). Although these Stokes lines do not intersect the real axis for finite $s$, the Stokes lines have a width which grows linearly in $s$ (away from the generating singularity). Thus for large $s$ the parallel Stokes lines will eventually impinge on the real axis, and will affect the behaviour of the solution at infinity (see King (1998) for an example of this phenomenon in an o.d.e.). This corresponds to rescaling for large $s$ in the integrals $I_{t 1}$ and $I_{t 2}$ and repeating the steepest descent analysis.

Neither of these issues is central to the analysis in this paper, which is concerned with the Stokes line structure for $s$ and $t$ of $\mathcal{O}(1)$, and we will not consider them further.

\section{(c) The Borel plane}

The Stokes structure determined in the previous section can be conveniently illustrated in terms of the Borel plane. Such an approach has been taken in Howls et al. (2005), to which the interested reader is referred for the details. Here we merely give a sketch of the behaviour for illustrative purposes.

In the Borel plane the integrals are of the form

$$
\int_{0}^{\infty} e^{-z / \epsilon} F(z, s, t) \mathrm{d} z,
$$




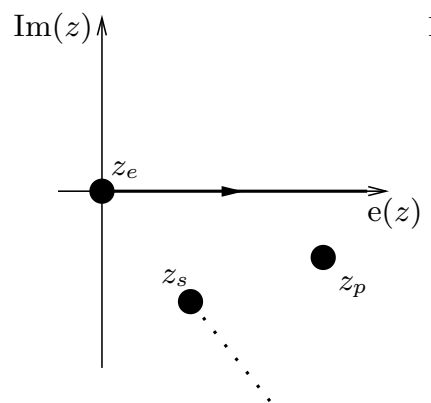

(a)

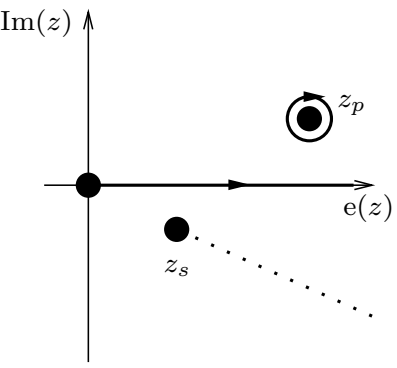

(b)

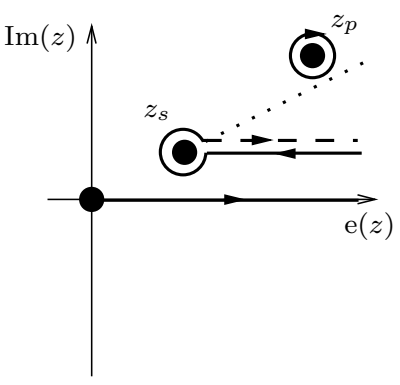

(c)

Figure 7. The Borel plane for $t<1 / \sqrt{3}$; (a) $s<0$, (b) $0<s<1 / \sqrt{3}-t$, (c) $s>1 / \sqrt{3}-t$. The solid contours are on the upper Riemann sheet, while the dashed contours lie on the lower Riemann sheet.

along the real $z$ axis. We will focus on $I_{t}$, for which $z=p(1+i s)+i p\left(1-p^{2}\right) t$. The endpoint corresponds to $z=z_{e}=0$, the pole to $z=z_{p}=\left.z\right|_{p=1}$ and the saddle to $z=z_{s}=\left.z\right|_{p=p_{s}}$ where $p=p_{s}$ is the solution of $\mathrm{d} z / \mathrm{d} p=0$. The point $z=z_{s}$ is a square root branch point of $f$ so that the relevant section of the Borel plane is a two sheeted Riemann surface, cut from $z_{s}$ to infinity along $\arg (z)=\arg \left(z_{s}\right)$. The higher-order Stokes phenomenon (which we will describe in Section c) occurs when the three points $z_{e}, z_{p}$ and $z_{s}$ are colinear, and $z_{p}$ passes from one sheet of the Riemann surface to another. The steepest descent paths in the Borel plane lie parallel to the real axis. The endpoint is always the dominant contribution. Unless $t>0$ is small or $|s|$ is large the largest subdominant exponential comes from the saddle point, and the pole contribution is subdominant with respect to the saddle point contribution, i.e. $\operatorname{Re}\left(z_{s}\right)<\operatorname{Re}\left(z_{p}\right)$. We assume that $s$ and $t$ are such that this is the case.

Let us now look at the positions of $z_{s}$ and $z_{p}$ as $s$ increases from $-\infty$ to $\infty$ along the real axis for fixed $t$.

For $t<1 / \sqrt{3}$, starting with large negative $s$ we have $\arg z_{s}<\arg z_{p}<0$ (Figure $7 \mathrm{a})$. As $s$ increases $\arg z_{s}$ and $\arg z_{p}$ increase and at $s=0, z_{p}$ crosses the real axis, and therefore the integration contour from the endpoint (Figure $7 \mathrm{~b}$ ). This switches on the pole contribution. When $s=1 / \sqrt{3}-t, z_{s}$ crosses the real axis. Since the saddle point is a branch point of the Riemann surface, the integration to infinity on the real axis is now on the wrong sheet, and we need to integrate back around the branch point by integrating from $\infty+i \arg \left(z_{s}\right)$ to $z_{s}$ as illustrated in Figure 7c. This extra integration is the saddle point contribution.

For $t>1 / \sqrt{3}$, starting with large negative $s$ we again have $\arg z_{s}<\arg z_{p}<0$ (Figure 8a). Now as we increase $s$, when $s$ reaches $s_{1}$, the smallest root of (3.20), $\arg \left(z_{p}-z_{s}\right)=0$. Since $\operatorname{Re}\left(z_{p}\right)>\operatorname{Re}\left(z_{s}\right)$ the pole crosses the branch cut in the Riemann surface and moves onto the lower Riemann sheet, as illustrated in Figure 8b. As $s$ increases further $\arg z_{s}$ and $\arg z_{p}$ continue to increase and this time $\arg z_{s}$ reaches zero first, at $s=1 / \sqrt{3}-t<0$. We now have an integration path around the branch point along $\operatorname{Im}(z)=\operatorname{Im}\left(z_{s}\right)$ as above (Figure 8c).

Now when $s=0, z_{p}$ crosses the real axis. But because the integration contour from $z_{e}$ is on the upper Riemann sheet, and $z_{p}$ is on the lower Riemann sheet, it 


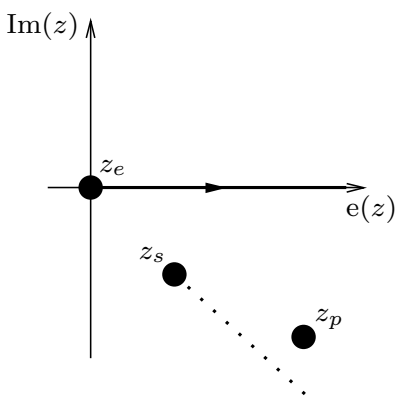

(a)

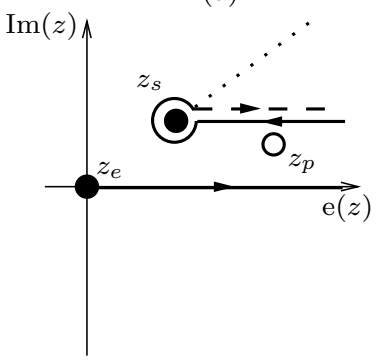

(d)

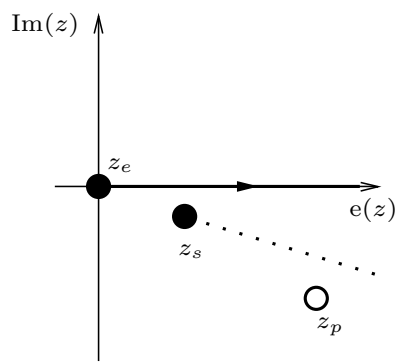

(b)

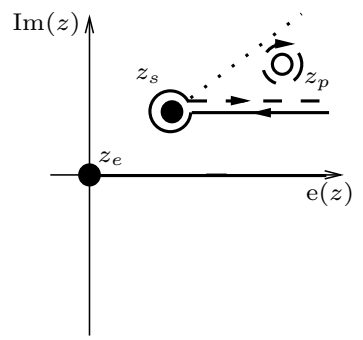

(e)

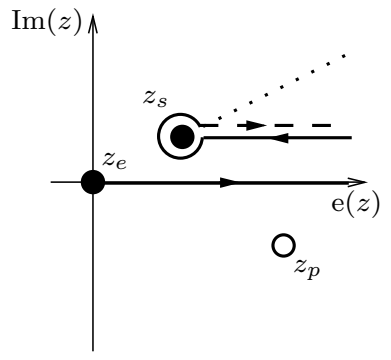

(c)

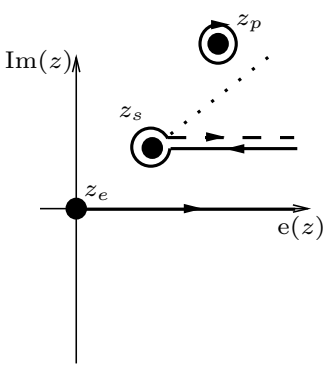

(f)

Figure 8. The Borel plane for $t>1 / \sqrt{3}$; (a) $s<s_{1}$, (b) $s_{1}<s<1 / \sqrt{3}-t$, (c) $1 / \sqrt{3}-t<s<0$, (d) $0<s<s_{2}$, (e) $s_{2}<s<s_{3}$, (f) $s>s_{3}$. The solid contours are on the upper Riemann sheet, while the dashed contours lie on the lower Riemann sheet. Where $z_{p}$ is shown as a solid dot it lies on the upper Riemann sheet; where it is shown hollow it lies on the lower Riemann sheet.

does not cross the integration contour, but passes "underneath" it. Thus no pole contribution is turned on (Figure $8 \mathrm{~d}$ ).

As $s$ increases further $\operatorname{Im}\left(z_{p}-z_{s}\right)$ increases, until at $s=s_{2}$ say $\operatorname{Im}\left(z_{p}\right)=\operatorname{Im}\left(z_{s}\right)$ and the pole crosses the new integration contour around the branch point. This is the saddle point turning on the pole contribution (Figure 8e).

As $s$ increases further $\arg \left(z_{p}-z_{s}\right)$ increases until at $s=s_{3}$, the second root of (3.20), $\arg \left(z_{p}-z_{s}\right)=0$ and $z_{p}$ passes back through the branch point to reemerge on the top Riemann sheet (Figure 8f). The pole has then effectively emerged on the other side of the line of integration $0 \leq \operatorname{Re}(z) \leq \infty, \operatorname{Im}(z)=0$, without actually crossing it.

The solution of (1.10)-(1.11) by Fourier transform, and the interpretation of the resulting integrals in the Borel plane works exceedingly well for this problem. However, while this approach is very powerful when applicable, for non-constantcoefficient linear equations the equation for the Fourier transform of the solution will not be solvable in general, while for nonlinear equations the Fourier transform itself is not applicable. We expect nonlinear equations such as (1.6) to exhibit similar patterns of Stokes switching, and we wish to develop techniques for determining the Stokes structure of such problems.

While an integral representation of the solution will not be available in general, the asymptotic expansion of the dominant exponential is available, even for nonlinear problems. Our aim in Sections 3-5 is to see if we can recover the Stokes structure 
illuminated in Section 2 without using the integral representation of the solution, but working directly with the asymptotic series for the dominant exponential. As well as giving a different interpretation of higher-order Stokes phenomenon, such techniques may be more readily applicable to nonlinear problems.

\section{Asymptotic analysis of the equation directly}

Having determined the Stokes structure of the problem we shall now reproduce these results using perturbation expansions and optimal truncation, without using the integral formulation of the solution. As we have just discussed, the techniques of this and the following sections may be more readily applicable to nonlinear problems, where an integral formulation of the solution may not be readily available.

The first step in the procedure is to determine the asymptotic behaviour of the late terms in the asymptotic expansion of $\phi$, which we do in this section. The second step is to optimally truncate the series for $\phi$ and study the behaviour of the remainder, which we do in Section 4.

(a) A naïve perturbation expansion

We begin by seeking a naïve perturbation expansion

$$
\phi \sim \sum_{n=0}^{\infty} \epsilon^{2 n} \phi^{(n)} .
$$

Where we need to distinguish this dominant asymptotic series from the subdominant exponentials switched on across Stokes lines, we label this series $\phi_{e}$, since it corresponds to the endpoint contribution of the integral form of the solution. We find the leading-order equation

$$
\begin{aligned}
\phi_{t}^{(0)}-\phi_{s}^{(0)} & =-\frac{1}{1+s^{2}}, \\
\phi^{(0)}(s, 0) & =\tan ^{-1} s,
\end{aligned}
$$

and in general

$$
\begin{aligned}
\phi_{t}^{(n)}-\phi_{s}^{(n)} & =\phi_{s s s}^{(n-1)}, \\
\phi^{(n)}(s, 0) & =0
\end{aligned}
$$

for $n \geq 1$. Hence

$$
\phi^{(0)}=\tan ^{-1}(s)=-\frac{i}{2} \log (s-i)+\frac{i}{2} \log (s+i)+\frac{\pi}{2} .
$$

Now, much as we did in Section 2, we shall consider each of these leading-order solutions separately. This corresponds to decomposing

$$
\frac{1}{1+s^{2}}=\frac{i}{2(s+i)}-\frac{i}{2(s-i)},
$$

solving for each part separately, and then adding. Let us first consider

$$
\phi^{(0)}=-\frac{i}{2} \log (s-i) .
$$


In order to simplify our workings we transform to a moving frame by setting

$$
\tau=t, \quad \sigma=s+t-i .
$$

With this change of variables equation (1.10) becomes

$$
\phi_{\tau}=\epsilon^{2} \phi_{\sigma \sigma \sigma}+\frac{i}{2(\sigma-\tau)}
$$

while equations (3.4)-(3.5) become

$$
\begin{aligned}
\phi_{\tau}^{(n)} & =\phi_{\sigma \sigma \sigma}^{(n-1)} \\
\phi^{(n)}(\sigma, 0) & =0
\end{aligned}
$$

with

$$
\phi^{(0)}=-\frac{i}{2} \log (\sigma-\tau)
$$

Our task is to find the Stokes line structure. Following Dingle (1973) we know that we can find the Stokes lines if we know the form of the late terms in the expansion (3.1).

\section{(b) Finding the late terms}

Having found the leading-order term $\phi^{(0)}$ in our expansion we are able to recursively derive all the other terms $\phi^{(n)}$ using (3.9)-(3.10). We find

$$
\phi_{\tau}^{(1)}=\phi_{\sigma \sigma \sigma}^{(0)}=-\frac{i}{(\sigma-\tau)^{3}} .
$$

Hence

$$
\phi^{(1)}=-\frac{i}{2(\sigma-\tau)^{2}}+\frac{i}{2 \sigma^{2}} .
$$

We note that $\phi^{(0)}$ has a singularity at $\sigma-\tau=0$. As expected we see that the first order term $\phi^{(1)}$ preserves this singularity. In addition, however, it also has a singularity $\dagger$ at $\sigma=s+t-i=0$. We find that at later stages both singularities are preserved but no further singularities are created. At the next order

$$
\phi_{\tau}^{(2)}=\phi_{\sigma \sigma \sigma}^{(1)}=\frac{i 4 !}{2(\sigma-\tau)^{5}}-\frac{i 4 !}{2 \sigma^{5}}
$$

so that

$$
\phi^{(2)}=\frac{i 3 !}{2(\sigma-\tau)^{4}}-\frac{i 3 !}{2 \sigma^{4}}\left(1+\frac{4 \tau}{\sigma}\right)
$$

$\uparrow$ The fact that both singularities are not in the leading-order solution is due to the choice of initial condition — putting $\phi(\sigma, \tau=0)=0$ would result in both singularities being present in $\phi^{(0)}$. 
In general the $n^{\text {th }}$ term in the expansion is

$$
\phi^{(n)}=\frac{i(-1)^{n}(2 n-1) !}{2(\sigma-\tau)^{2 n}}+\frac{i(-1)^{n+1}(2 n-1) !}{2 \sigma^{2 n}} \sum_{j=0}^{n-1}\left(\frac{\tau}{\sigma}\right)^{j} b_{n, j},
$$

where $b_{n, j}$ satisfies the recurrence relation

$$
\begin{aligned}
b_{n, j+1}(j+1)(2 n-1)(2 n-2) & =(2 n-2+j)(2 n-1+j)(2 n+j) b_{n-1, j}, \\
b_{n, 0} & =1
\end{aligned}
$$

that is

$$
b_{n, j}=\frac{(2 n-1+j) !}{(2 n-1) ! j !} .
$$

The series (3.1), (3.13) corresponds to the full asymptotic expansion of the endpoint contribution in the integral formulation of Section 2.

In both linear (Dingle (1973)) and nonlinear (Chapman et al. (1998)) singularly perturbed o.d.e. problems we find the $n^{\text {th }}$ term of the expansion to be a single term with the form of 'factorial over power'. In this case, however, we have a sum of such terms. We shall approximate this sum as $n \rightarrow \infty$, after which we will be in a similar position to the one faced when tackling an o.d.e., and we will be able to describe the Stokes switching.

Our first task is therefore to asymptotically evaluate the sum

$$
I=\sum_{j=0}^{n-1}\left(\frac{\tau}{\sigma}\right)^{j} \frac{(2 n-1+j) !}{(2 n-1) ! j !}
$$

for large $n$. We begin by rewriting the sum as an integral

$$
I=1+\frac{1}{2 \pi i} \oint_{C}\left(-\frac{\tau}{\sigma}\right)^{z} \frac{\Gamma(2 n+z)}{\Gamma(2 n) \Gamma(z+1)} \frac{\pi}{\sin \pi z} \mathrm{~d} z
$$

where the contour $C$ is shown in Figure 9.

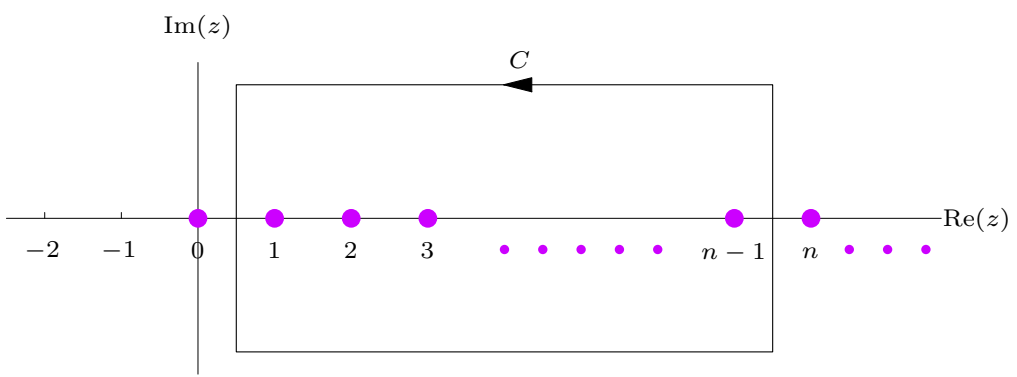

Figure 9. Contour of integration for $I$. Dots indicate poles.

Setting $z=n v$ and using Stirling's formula, $\Gamma(n) \sim \sqrt{2 \pi} n^{n-1 / 2} \mathrm{e}^{-n}$, we find

$$
\frac{\Gamma(2 n+z)}{\Gamma(2 n) \Gamma(z+1)} \sim \frac{1}{\sqrt{\pi}} \frac{(2+v)^{2 n+v n}}{2^{2 n} v^{v n}} \frac{1}{(2+v)^{1 / 2}(n v)^{1 / 2}},
$$


as $n \rightarrow \infty$, for $\operatorname{Re}(v)>-2$. Since

$$
\frac{1}{\sin \pi v n} \sim \begin{cases}-2 i \mathrm{e}^{i \pi v n} & \text { for } \operatorname{Im}(v)>0 \\ 2 i \mathrm{e}^{-i \pi v n} & \text { for } \operatorname{Im}(v)<0\end{cases}
$$

we find that away from the real axis

$$
I \sim 1+\int_{C} F(v ; \tau, \sigma, n) \mathrm{e}^{n f(v ; \tau, \sigma)} \mathrm{d} v
$$

where

$$
\begin{gathered}
f(v ; \tau, \sigma)= \begin{cases}(2+v) \log (2+v)-2 \log 2+v \log \left(-\frac{\tau}{\sigma v}\right)+i \pi v, & \operatorname{Im}(v)>0 \\
(2+v) \log (2+v)-2 \log 2+v \log \left(-\frac{\tau}{\sigma v}\right)-i \pi v, & \operatorname{Im}(v)<0\end{cases} \\
F(v ; \tau, \sigma, n)= \begin{cases}-\frac{n^{1 / 2}}{\sqrt{\pi}(2+v)^{1 / 2} v^{1 / 2}}, & \operatorname{Im}(v)>0, \\
\frac{n^{1 / 2}}{\sqrt{\pi}(2+v)^{1 / 2} v^{1 / 2}}, & \operatorname{Im}(v)<0,\end{cases}
\end{gathered}
$$

and the branch of $\log (-\tau / \sigma)$ is such that the imaginary part is $-\pi$ when $\tau$ and $\sigma$ are real. We now deform the contour to the steepest descent contour (making sure we still go through the "endpoint" $v=1$, so that we do not pick up any more of the poles on the real axis). There is one saddle point, which may or may not be on the integration contour, depending on the value of $\tau / \sigma$. Two typical contours are shown in Figure 10. Note that there are branch points at $v=0$ and $v=-2$, and poles on the real axis at $z=k$ and $z=-2 n-k, k=0,1, \cdots$. The deformed contour crosses the real axis with $-2<v<0$, so that we don't pick up any extra pole contributions, and the branch cut from $v=-2$ has no effect. There are thus three contributions possible in the evaluation of the integral: the saddle point, the integral around the branch cut from $v=0$, and the integral through the "endpoint" $v=1$.

The saddle point integral, $C_{1}$

The position of the saddle point is given by

$$
f^{\prime}\left(v_{s}\right)=\log \left(\frac{\left(2+v_{s}\right)}{v_{s}} \frac{\tau}{\sigma}\right)=0, \quad \text { so that } \quad v_{s}=\frac{2 \tau}{(\sigma-\tau)}=\frac{2 t}{s-i}
$$

If $s, t \in \mathbb{R}$ with $t>0$ then $\operatorname{Im}\left(v_{s}\right)>0$ and the saddle lies in the upper half plane, as shown in Figure 10. A standard application of the method of steepest descents shows the leading-order contribution from the saddle point is given by

$$
-\sqrt{\frac{2 \pi}{-n f^{\prime \prime}\left(v_{s}\right)}} F\left(v_{s}\right) \mathrm{e}^{n f\left(v_{s}\right)}=\left(\frac{\sigma}{\sigma-\tau}\right)^{2 n} .
$$

Note that this contribution to $I$ is equal and opposite to the first term in (3.13). 

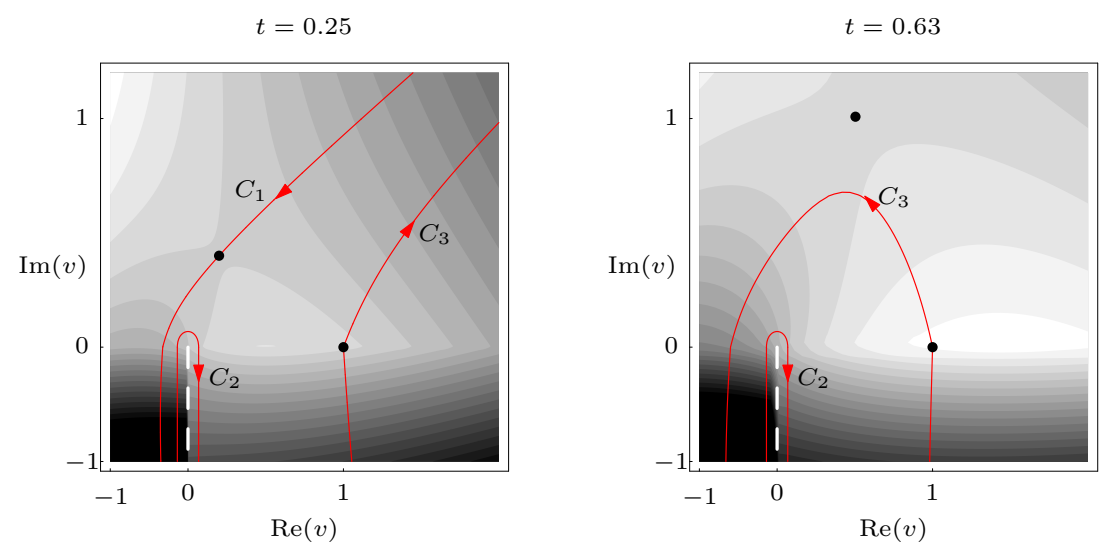

Figure 10. Contours of integration for $I$ at $s=0.5$ as $t$ varies. Branch cuts shown dashed and chosen to best illustrate contours. Dots indicate saddle and end points.

\section{The branch-cut integral, $C_{2}$}

The point $v=0$ is a branch point only of the asymptotic approximation of the integrand as $n \rightarrow \infty$ away from the real axis, not of the integrand itself. If we return to the original integrand along $C_{2}$ we can deform safely to infinity, picking up a single contribution from the pole at the origin, which is -1 (since the integral is clockwise). This cancels the 1 in (3.17).

The end-point integral, $C_{3}$

The integral decays rapidly away from the end-point. The approximation (3.18) is not valid in the vicinity of the real axis, so we must return to the original integrand (3.17). Letting $z=n+u$ we must evaluate

$$
\begin{aligned}
I_{C_{3}} & =\frac{1}{2 \pi i} \int_{-1 / 2-i \infty}^{-1 / 2+i \infty}\left(-\frac{\tau}{\sigma}\right)^{(n+u)} \frac{\Gamma(3 n+u)}{\Gamma(2 n) \Gamma(n+1+u)} \frac{\pi}{\sin \pi(n+u)} \mathrm{d} u, \\
& =\frac{1}{2 \pi i} \int_{-1 / 2-i \infty}^{-1 / 2+i \infty}\left(-\frac{\tau}{\sigma}\right)^{(n+u)} \frac{\Gamma(3 n+u)}{\Gamma(2 n) \Gamma(n+1+u)} \frac{(-1)^{n} \pi}{\sin \pi u} \mathrm{~d} u .
\end{aligned}
$$

Expanding for large $n$ using Stirling's formula we find

$$
I_{C_{3}} \sim \frac{1}{2 i \sqrt{3 \pi n}}\left(\frac{27 \tau}{4 \sigma}\right)^{n} \int_{-1 / 2-i \infty}^{-1 / 2+i \infty}\left(-\frac{3 \tau}{\sigma}\right)^{u} \frac{1}{\sin \pi u} \mathrm{~d} u .
$$

If $|3 \tau / \sigma|<1$ we close the contour to the right and evaluate via a sum of residues from the poles at $u=0,1, \cdots$. Noting that the poles are encircled clockwise we find

$$
I_{C_{3}} \sim-\frac{1}{\sqrt{3 \pi n}}\left(\frac{27 \tau}{4 \sigma}\right)^{n} \sum_{k=0}^{\infty}\left(\frac{3 \tau}{\sigma}\right)^{k}=\frac{1}{\sqrt{3 \pi n}}\left(\frac{27 \tau}{4 \sigma}\right)^{n} \frac{\sigma}{(3 \tau-\sigma)}
$$


If $|3 \tau / \sigma|>1$ we must close the contour to the left, evaluating via a sum of residues from poles at $u=-1,-2, \cdots$ encircled anti-clockwise, giving

$$
I_{C_{3}} \sim \frac{1}{\sqrt{3 \pi n}}\left(\frac{27 \tau}{4 \sigma}\right)^{n} \sum_{k=1}^{\infty}\left(\frac{\sigma}{3 \tau}\right)^{k}=\frac{1}{\sqrt{3 \pi n}}\left(\frac{27 \tau}{4 \sigma}\right)^{n} \frac{\sigma}{(3 \tau-\sigma)} .
$$

Thus, in fact, the approximation is the same whatever the value of $|3 \tau / \sigma|$.

\section{(c) Higher-order Stokes line}

Now let us see when the saddle point contributes to the approximation of the integral $I$. There is a Stokes line when the contour through the endpoint $C_{3}$ goes through the saddle point. This Stokes line is given by

$$
\operatorname{Im}(f(1))=\operatorname{Im}\left(f\left(v_{s}\right)\right), \quad \text { i.e. } \operatorname{Im}\left(\log \left(\frac{\tau}{\sigma}\right)\right)=\operatorname{Im}\left(\log \left(\frac{\sigma}{\sigma-\tau}\right)^{2}\right)
$$

For $s$ and $t$ real this implies

$$
1+s^{4}-3 t^{2}-2 s t^{3}+s^{2}\left(2-3 t^{2}\right)=0 .
$$

Thus the saddle point only contributes when $t$ and $s$ are such that we are at a point below this curve, which is shown in Figure 11. This Stokes line is not a Stokes line in the approximation of the solution of the original p.d.e., which we examined in $\S 2$ and across which $\phi_{e}$ turns on $\phi_{s}$ and $\phi_{p}$; rather it is a Stokes line in the approximation of the late terms in the full expansion of the dominant contribution $\phi_{e}$. We shall call such a Stokes line a higher-order Stokes line. Let us see how condition (3.19)

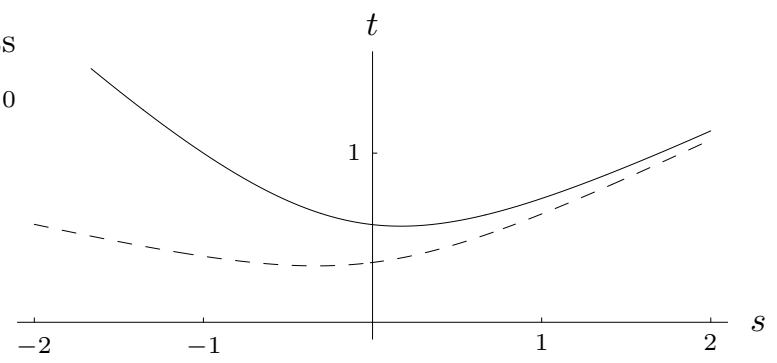

Figure 11. Higher order Stokes line (solid) and higher order anti-Stokes line (dashed) in real space.

relates to the transition between Fig $8(\mathrm{e})$ and $8(\mathrm{f})$, where $z_{e}, z_{s}$ and $z_{p}$ are colinear. The saddle-point contribution to $I$ is of the form

$$
K_{s}(2 n-1) !\left(-\frac{e^{f\left(v_{s}\right)}}{\sigma^{2}}\right)^{n}
$$

while the end-point contribution to $I$ is of the form

$$
K_{e} n^{-1 / 2}(2 n-1) !\left(-\frac{e^{f(1)}}{\sigma^{2}}\right)^{n},
$$


where $K_{s}$ and $K_{e}$ do not depend on $n$ to leading order. When these late terms are Borel summed the corresponding singularities in the Borel plane are

$$
z_{p}=\frac{i e^{f\left(v_{s}\right) / 2}}{\sigma} \quad \text { and } \quad z_{s}=\frac{i e^{f(1) / 2}}{\sigma}
$$

respectively. These are colinear with $z_{e}=0$ when $\arg \left(z_{p}\right)=\arg \left(z_{s}\right)$, i.e.

$$
\arg \left(\frac{i e^{f\left(v_{s}\right) / 2}}{\sigma}\right)=\arg \left(\frac{i e^{f(1) / 2}}{\sigma}\right)
$$

which is equivalent to (3.19).

Returning to approximation of the integral $I$, we find that there is an antiStokes line when the contribution from the endpoint $C_{3}$ and the contribution from the saddle point change in dominance. Again, this is not an anti-Stokes line of the approximation of the original p.d.e., which would occur when $\phi_{s}$ and $\phi_{p}$ change in dominance, but an anti-Stokes line in the approximation of the late terms of the full expansion of $\phi_{e}$. This higher order anti-Stokes line is given by

$$
\operatorname{Re}(f(1))=\operatorname{Re}\left(f\left(v_{s}\right)\right), \quad \text { i.e. } \operatorname{Re}\left(\log \left(\frac{27 \tau}{\sigma}\right)\right)=\operatorname{Re}\left(\log \left(\frac{4 \sigma^{2}}{(\sigma-\tau)^{2}}\right)\right)
$$

The saddle point contribution is dominant when $t$ and $s$ are such that we are at a point below the dashed curve shown in Figure 11.

Combining the expressions for $I_{C_{1}}$ and $I_{C_{3}}$ with (3.13) we have

$$
\phi^{(n)} \sim \begin{cases}\frac{i(-1)^{n}(2 n-1) !}{2(\sigma-\tau)^{2 n}}-\frac{i \sigma(-1)^{n}(2 n-1) !}{2 \sqrt{3 \pi n}(3 \tau-\sigma)}\left(\frac{27 \tau}{4 \sigma^{3}}\right)^{n} & \text { above the Stokes line } \\ -\frac{i \sigma(-1)^{n}(2 n-1) !}{2 \sqrt{3 \pi n}(3 \tau-\sigma)}\left(\frac{27 \tau}{4 \sigma^{3}}\right)^{n} & \text { below the Stokes line }\end{cases}
$$

We write

$$
\begin{aligned}
\phi^{(n)}=\phi_{e 1}^{(n)} & \sim \mathcal{S} \frac{i(-1)^{n}(2 n-1) !}{2(\sigma-\tau)^{2 n}}-\frac{i \sigma(-1)^{n}(2 n-1) !}{2 \sqrt{3 \pi n}(3 \tau-\sigma)}\left(\frac{27 \tau}{4 \sigma^{3}}\right)^{n} \\
& =\mathcal{S} \phi_{1}^{(n)}+\phi_{2}^{(n)}
\end{aligned}
$$

where the Stokes multiplier $\mathcal{S}$ is zero below the Stokes line and unity above it. We emphasize here that the asymptotic approximation in (3.22) is as $n \rightarrow \infty$, and that the Stokes switching in $\phi^{(n)}$ refers to this limit, in contrast to the Stokes switching of $\phi$, which refers to the limit $\epsilon \rightarrow 0$ (remember also that $\phi^{(n)}$ is independent of $\epsilon$ ). Note also that $\phi_{1}^{(n)}$ and $\phi_{2}^{(n)}$ are only defined by (3.23) in the limit as $n \rightarrow \infty$. To allow us to define our truncation procedure we write $\phi=\phi_{1}+\phi_{2}$, with $\phi^{(n)}=\phi_{1}^{(n)}+\phi_{2}^{(n)}$ for all $n$, where the definition of $\phi_{1}^{(n)}$ and $\phi_{2}^{(n)}$ is arbitrary, except in the limit of large $n$.

\section{Stokes smoothing}

Having determined the form of the late terms of the asymptotic series of $\phi$, we can now truncate the series and study the behaviour of the remainder as in Daalhuis 
et al. (1995); Chapman et al. (1998). In this way we will observe the Stokes switching directly through a smoothing of the Stokes discontinuities.

We consider the tails of the series $\phi_{1}^{(n)}$ and $\phi_{2}^{(n)}$ in (3.22) separately. We shall truncate each optimally and examine the remainder as $n \rightarrow \infty$.

(a) Stokes smoothing for $\phi_{1}$

We write

$$
\phi_{1}=\sum_{n=0}^{N-1} \phi_{1}^{(n)} \epsilon^{2 n}+R_{1}
$$

At the optimal truncation point

$$
\left|\frac{\phi_{1}^{(N+1)} \epsilon^{2 N+2}}{\phi_{1}^{(N)} \epsilon^{2 N}}\right| \sim \frac{4 \epsilon^{2} N^{2}}{|\sigma-\tau|^{2}} \sim 1,
$$

so that $N \sim|\sigma-\tau| / 2 \epsilon$. From Dingle (1973) we know that a Stokes line is characterised by successive late terms in a series having the same phase. Thus we expect a Stokes line when $-(\sigma-\tau)^{2}=-(s-i)^{2}$ is real and positive, that is, when $s$ is pure imaginary. Putting the expansion (4.1) into (1.10) (and dropping the subscript) gives

$$
R_{1 \tau}-\epsilon^{2} R_{1 \sigma \sigma \sigma} \sim \phi_{\sigma \sigma \sigma}^{(N-1)} \epsilon^{2 N} \sim \frac{i(-1)^{N}(2 N) !}{2(\sigma-\tau)^{2 N+1}} \epsilon^{2 N}
$$

Motivated by the form of the right-hand side we write

$$
R_{1}=A(\sigma, \tau) \mathrm{e}^{-i(\sigma-\tau) / \epsilon} .
$$

Then as $\epsilon \rightarrow 0$ and $N \rightarrow \infty$

$$
A_{\tau}+3 A_{\sigma} \sim \frac{i(-1)^{N}(2 N) !}{2(\sigma-\tau)^{2 N+1}} \epsilon^{2 N} \mathrm{e}^{i(\sigma-\tau) / \epsilon} .
$$

We now define $u=\sigma-\tau$, choose $v$ so that $v_{\tau}+3 v_{\sigma}=0$, and change coordinates from $\sigma$ and $\tau$ to $u$ and $v$. Then

$$
2 A_{u} \sim \frac{i(-1)^{N}(2 N) !}{2 u^{2 N+1}} \epsilon^{2 N} \mathrm{e}^{i u / \epsilon} .
$$

Putting $u=r \mathrm{e}^{i \theta}$, with $N=r / 2 \epsilon+a$ where $a$ is bounded as $\epsilon \rightarrow 0$, and writing

$$
\frac{\partial}{\partial u}=-\frac{i \mathrm{e}^{-i \theta}}{r} \frac{\partial}{\partial \theta}
$$

gives

$$
A_{\theta} \sim-\sqrt{\frac{\pi r}{8 \epsilon}} \exp \left[(-i \pi-2 i \theta)\left(\frac{r}{2 \epsilon}+a\right)-\frac{r}{\epsilon}+\frac{i r \mathrm{e}^{i \theta}}{\epsilon}\right]
$$


The right-hand side is exponentially small except near the Stokes line $\theta=-\pi / 2$. We rescale in the vicinity of the Stokes line by setting $\theta=-\pi / 2+\sqrt{\epsilon} \bar{\theta}$ to give

$$
A_{\bar{\theta}} \sim-\sqrt{\frac{\pi r}{8}} \exp \left(-\frac{r \bar{\theta}^{2}}{2}\right) .
$$

Since $A \rightarrow 0$ as $\bar{\theta} \rightarrow-\infty$ we have

$$
A \sim-\frac{\sqrt{\pi}}{2} \int_{-\infty}^{\bar{\theta} \sqrt{r / 2}} \mathrm{e}^{-x^{2}} \mathrm{~d} x .
$$

Matching as $\bar{\theta} \rightarrow+\infty$ we find that the jump in the Stokes multiplier across the Stokes line is $-\pi / 2$. Thus, across the Stokes line where $\operatorname{Re}(s)=0$,

$$
\phi_{p 1}=-\frac{\pi}{2} \mathrm{e}^{-i(\sigma-\tau) / \epsilon}=-\frac{\pi}{2} \mathrm{e}^{-(1+i s) / \epsilon},
$$

is switched on.

Thus we have been able to observe directly the switching on of the pole contribution by the endpoint.

(b) Stokes smoothing for $\phi_{2}$

We now consider

$$
\phi_{2}^{(n)}=\frac{i(-1)^{n}(2 n-1) !}{2 \sqrt{3 \pi n}}\left(\frac{27 \tau}{4 \sigma^{3}}\right)^{n} \frac{\sigma}{3 \tau-\sigma} .
$$

We expect a Stokes line when successive terms have the same phase, i.e. when $\sigma^{3} / \tau$ is real and positive. This corresponds to $t=1 / \sqrt{3}-\operatorname{Re}(s)$, or $\operatorname{Im}(s)=1$. As before we set

$$
\phi_{2}=\sum_{n=0}^{N-1} \phi_{2}^{(n)} \epsilon^{2 n}+R_{2}
$$

At the optimal truncation point

$$
\left|\frac{\phi_{2}^{(N+1)} \epsilon^{2 N+2}}{\phi_{2}^{(N)} \epsilon^{2 N}}\right| \sim 4 \epsilon^{2} N^{2}\left|\frac{27 \tau}{4 \sigma^{3}}\right| \sim 1
$$

so that $N \sim\left|4 \sigma^{3} / 27 \tau\right|^{1 / 2} / 2 \epsilon$. Equation (1.10) becomes

$$
R_{2 \tau}-\epsilon^{2} R_{2 \sigma \sigma \sigma} \sim \frac{i(-1)^{N}(2 N) !}{4 \sqrt{3 \pi N}}\left(\frac{27 \tau}{4 \sigma^{3}}\right)^{N} \frac{\sigma \epsilon^{2 N}}{3 \tau^{2}-\tau \sigma} .
$$

Motivated by the right-hand side we set

$$
R_{2}=A(\sigma, \tau) \mathrm{e}^{-i u / \epsilon} .
$$

where

$$
u=\sqrt{\frac{4 \sigma^{3}}{27 \tau}}
$$

Article submitted to Royal Society 
Then as $\epsilon \rightarrow 0$ and $N \rightarrow \infty$

$$
A_{\tau}+3 u_{\sigma} u_{\sigma \sigma} A+3\left(u_{\sigma}\right)^{2} A_{\sigma}=A_{\tau}+\frac{\sigma}{\tau} A_{\sigma}+\frac{A}{2 \tau} \sim \frac{i(-1)^{N}(2 N) !}{4 \sqrt{3 \pi N} u^{2 N}} \frac{\sigma \epsilon^{2 N} \mathrm{e}^{i u / \epsilon}}{\left(3 \tau^{2}-\tau \sigma\right)} .
$$

The homogeneous form of the first order p.d.e. (4.14) has solutions of the form

$$
\frac{1}{\sqrt{\tau}} f\left(\frac{\tau}{\sigma}\right)
$$

We therefore set $v=\tau / \sigma$, and change variables to $u$ and $v$, writing

$$
A=\frac{1}{\sqrt{\tau}} B(u, v) .
$$

Noting that

$$
\begin{aligned}
\partial_{\sigma} & =u_{\sigma} \partial_{u}+v_{\sigma} \partial_{v}=\frac{3 u}{2 \sigma} \partial_{u}-\frac{\tau}{\sigma^{2}} \partial_{v}, \\
\partial_{\tau} & =u_{\tau} \partial_{u}+v_{\tau} \partial_{v}=-\frac{u}{2 \tau} \partial_{u}+\frac{1}{\sigma} \partial_{v},
\end{aligned}
$$

we find

$$
\frac{u}{\tau^{3 / 2}} B_{u} \sim \frac{i(-1)^{N}(2 N) !}{4 \sqrt{3 \pi N} u^{2 N}} \frac{\sigma \epsilon^{2 N}}{\left(3 \tau^{2}-\tau \sigma\right)} \mathrm{e}^{i u / \epsilon}
$$

Noting that

$$
\frac{\sigma \tau^{3 / 2}}{3 \tau^{2}-\tau \sigma}=\frac{u^{1 / 2} v^{3 / 4} 3^{3 / 4}}{2^{1 / 2}(3 v-1)}
$$

we have

$$
B_{u} \sim \frac{i 3^{1 / 4}(-1)^{N}(2 N) ! \epsilon^{2 N} \mathrm{e}^{i u / \epsilon}}{2^{5 / 2} \sqrt{\pi N} u^{2 N+1 / 2}} \frac{v^{3 / 4}}{(3 v-1)}
$$

Putting $u=r \mathrm{e}^{i \theta}$ with $N=r / 2 \epsilon+a$ as before gives

$$
B_{\theta} \sim-\frac{r^{1 / 2} \mathrm{e}^{i \theta / 2} 3^{1 / 4} v^{3 / 4}}{2^{3 / 2}(3 v-1)} \exp \left((-i \pi-2 i \theta)\left(\frac{r}{2 \epsilon}+a\right)-\frac{r}{\epsilon}+\frac{i r e^{i \theta}}{\epsilon}\right) .
$$

The right-hand side is exponentially small except at the Stokes line $\theta=-\pi / 2$. Rescaling near the Stokes line by setting $\theta=-\pi / 2+\sqrt{\epsilon} \bar{\theta}$ gives

$$
B_{\bar{\theta}} \sim-\frac{\epsilon^{1 / 2} r^{1 / 2} \mathrm{e}^{-i \pi / 4} 3^{1 / 4} v^{3 / 4}}{2^{3 / 2}(3 v-1)} \exp \left(-\frac{r \bar{\theta}^{2}}{2}\right)
$$

Since $B \rightarrow 0$ as $\bar{\theta} \rightarrow-\infty$ we have

$$
B \sim-\frac{\mathrm{e}^{-i \pi / 4} 3^{1 / 4} v^{3 / 4} \epsilon^{1 / 2}}{2(3 v-1)} \int_{-\infty}^{\bar{\theta} \sqrt{r / 2}} \mathrm{e}^{-x^{2}} \mathrm{~d} x
$$

Article submitted to Royal Society 
Matching as $\bar{\theta} \rightarrow+\infty$ and recalling that $A=B / \sqrt{\tau}$ we find that the jump in the Stokes multiplier across the Stokes line is

$$
-\frac{\sqrt{\pi} \mathrm{e}^{-i \pi / 4} 3^{1 / 4} v^{3 / 4} \epsilon^{1 / 2}}{2(3 v-1) \tau^{1 / 2}} .
$$

Thus, across the Stokes line where $t=1 / \sqrt{3}-\operatorname{Re}(s)$,

$$
\begin{aligned}
\phi_{s 1}^{(0)} & =-\frac{\sqrt{\pi} \mathrm{e}^{-i \pi / 4} 3^{1 / 4} v^{3 / 4} \epsilon^{1 / 2}}{2(3 v-1) \tau^{1 / 2}} \mathrm{e}^{-i u / \epsilon} \\
& =\frac{\sqrt{\pi} \mathrm{e}^{3 i \pi / 4} 3^{1 / 4} \tau^{1 / 4} \sigma^{1 / 4} \epsilon^{1 / 2}}{2(3 \tau-\sigma)} \exp \left(-\frac{i}{\epsilon}\left(\frac{4 \sigma^{3}}{27 \tau}\right)^{1 / 2}\right) \\
& =\frac{\sqrt{\pi} \mathrm{e}^{3 i \pi / 4} 3^{1 / 4} t^{1 / 4}(s+t-i)^{1 / 4} \epsilon^{1 / 2}}{2(2 t-s+i)} \exp \left(-\frac{i}{\epsilon}\left(\frac{4(s+t-i)^{3}}{27 t}\right)^{1 / 2}\right),
\end{aligned}
$$

is switched on.

Thus we have been able to observe directly the switching on of the saddle point contribution by the end point.

\section{(c) Second generation Stokes switching.}

From the analysis in Sections a and b we can start to construct Figure 6. So far we have determined the Stokes lines shown in Figure 12. Of course, by comparing

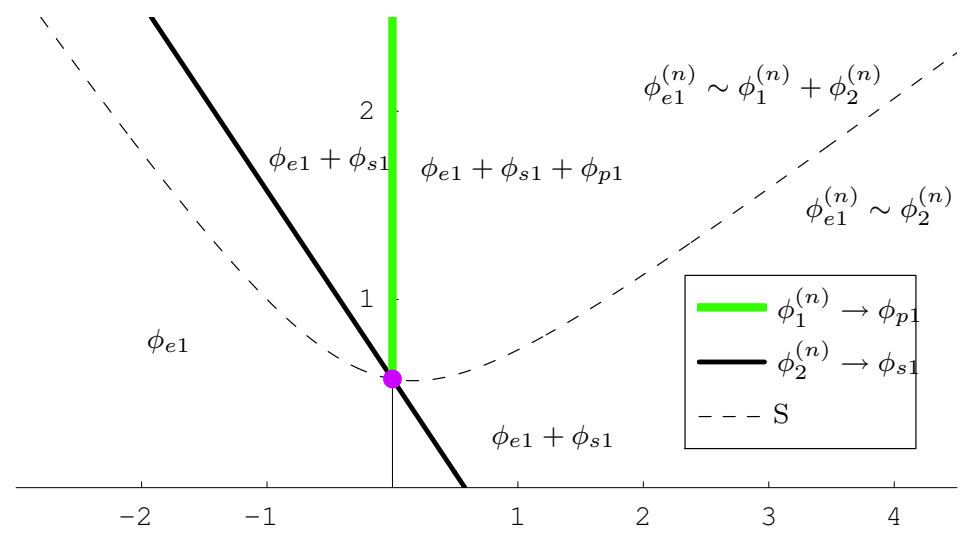

Figure 12. The Stokes lines across which the late terms in $\phi_{1}$ switch on $\phi_{p 1}$ and the late terms in $\phi_{2}$ switch on $\phi_{s 1}$. Also shown is the higher-order Stokes line (corresponding to Stokes multiplier $\mathcal{S}$ and shown dashed) across which the contribution $\phi_{1}^{(n)}$ disappears from the late terms of $\phi_{e 1}$.

with Figure 6 we know that Figure 12 is incomplete. However, we can see this even without Figure 6 to compare with, since as we circle the point $s=0, t=1 / \sqrt{3}$ we 
do not return to the solution we started with, and yet this point is not a branch point of the solution.

The missing Stokes line is that across which the saddle point switches on the pole. The original asymptotic series for $\phi(3.1)$, (3.13) cannot pick this up, since it corresponds to the endpoint contribution from the integral formulation. This extra Stokes line arises because, unlike $\phi_{p 1}, R_{2}=\phi_{s 1}^{(0)}$ (which corresponds to the saddle point contribution) does not satisfy the equation $R_{2 \tau}=\epsilon^{2} R_{2 \sigma \sigma \sigma}$ exactly, but only to the first two orders in $\epsilon$. Thus we have found only the leading term in the asymptotic expansion of $R_{2}$. This expansion is divergent, and will itself be responsible for turning on subdominant terms through Stokes phenomenon.

To see the saddle point contribution switch on the pole contribution we write

$$
R_{2}=\sum_{n=0}^{\infty} A(\sigma, \tau)^{(n)} \epsilon^{n+1 / 2} \mathrm{e}^{-i u / \epsilon}
$$

where

$$
A^{(0)}=\frac{\sqrt{\pi} \mathrm{e}^{3 i \pi / 4}(3 \sigma \tau)^{1 / 4}}{2(3 \tau-\sigma)} \quad \text { and } \quad u=\sqrt{\frac{4 \sigma^{3}}{27 \tau}} \text {. }
$$

Substituting into the equation $R_{2 \tau}=\epsilon^{2} R_{2 \sigma \sigma \sigma}$ we find

$$
A_{\tau}^{(n)}+\frac{\sigma}{\tau} A_{\sigma}^{(n)}+\frac{A^{(n)}}{2 \tau}=A_{\sigma \sigma \sigma}^{(n-2)}-\frac{i}{4 \sqrt{3 \sigma^{3} \tau}}\left(12 \sigma^{2} A_{\sigma \sigma}^{(n-1)}+6 \sigma A_{\sigma}^{(n-1)}-A^{(n-1)}\right),
$$

for $n \geq 1$ (with the convention that $A^{(-1)}=0$ ). A scaling analysis on (4.27) shows that $A^{(n)}$ will be of the form

$$
A^{(n)}=\frac{f_{n}(\sigma / \tau)}{\tau^{n+1 / 2}}
$$

giving

$$
\begin{aligned}
n f_{n} & =\frac{i \sqrt{3 \eta}}{2}\left(2 f_{n-1}^{\prime \prime}+\frac{f_{n-1}^{\prime}}{\eta}-\frac{f_{n-1}}{6 \eta^{2}}\right)-f_{n-2}^{\prime \prime \prime} \\
f_{0} & =\frac{\sqrt{\pi} e^{3 i \pi / 4}(3 \eta)^{1 / 4}}{2(3-\eta)}
\end{aligned}
$$

where $\eta=\sigma / \tau$, and ${ }^{\prime} \equiv \mathrm{d} / \mathrm{d} \eta$.

Although we cannot solve (4.29) exactly, to determine the Stokes lines we only need to know the late terms in the expansion. As $n \rightarrow \infty, f_{n}$ will diverge as factorial/power in the form

$$
f_{n} \sim \frac{F(\eta) \Gamma(n+\gamma(\eta))}{V(\eta)^{n+\gamma(\eta)}} .
$$

To leading-order as $n \rightarrow \infty$

$$
V=\left(V^{\prime}\right)^{3}+i \sqrt{3 \eta}\left(V^{\prime}\right)^{2} .
$$


Since the divergence is due to repeated differentiation of the singularity in $f_{0}$ at $\eta=3$, the boundary condition we must impose on $(4.32)$ is $V(3)=0$, giving

$$
V=i\left(\eta-1-\left(\frac{4 \eta^{3}}{27}\right)^{1 / 2}\right) .
$$

At the next order in $n\left(\mathcal{O}\left(f_{n} \log n\right)\right)$, we find that $\gamma^{\prime}=0$ so that $\gamma=$ constant. Then at $\mathcal{O}\left(f_{n}\right)$ we find an equation for $F$,

$$
(1-2 \gamma) F+2(\eta-3) F^{\prime}=0
$$

so that

$$
F=\Lambda(\eta-3)^{\gamma-1 / 2}
$$

where $\Lambda$ is constant. Now, as $\eta \rightarrow 3, V \sim-i(\eta-3)^{2} / 12$, so that $f_{n} \sim$ const. $(\eta-$ $3)^{-\gamma-1 / 2-2 n}$. For this to be consistent with $f_{0} \sim$ const. $(\eta-3)^{-1}$ requires $\gamma=1 / 2$.

To determine the constant $\Lambda$ we solve (4.29) in the limit $\eta \rightarrow 3$. With

$$
f_{n} \sim \frac{a_{n}}{(-i / 12)^{n+1 / 2}(\eta-3)^{2 n+1}},
$$

equations (4.29)-(4.30) become, at leading-order as $\eta \rightarrow 3$,

$$
\begin{aligned}
& a_{n}=(n-1 / 2) a_{n-1}, \\
& a_{0}=-\frac{i \sqrt{\pi}}{4} .
\end{aligned}
$$

Hence

$$
a_{n}=-\frac{i \Gamma(n+1 / 2)}{4}
$$

Comparing with (4.31) we see that $\Lambda=-i / 4$ and thus

$$
A^{(n)} \sim-\frac{i \Gamma(n+1 / 2)}{4 v^{n+1 / 2}} \quad \text { as } n \rightarrow \infty .
$$

where

$$
v=V \tau=i\left(\sigma-\tau-\left(\frac{4 \sigma^{3}}{27 \tau}\right)^{1 / 2}\right) .
$$

We expect there to be a Stokes line where successive terms have the same phase, i.e. where $v$ is real and positive. This corresponds to

$$
\frac{2}{\sqrt{27 t}} \operatorname{Im}\left(i(s+t-i)^{3 / 2}\right)=\operatorname{Im}(i s+1),
$$

such that $\operatorname{Im}(v)>0$. This is the 'missing' Stokes line from Figure 12. Note that since the series $R_{2}$ is only present to the right of the black line in Figure 12 then so is the 'missing', or what we shall call second-generation Stokes line $\dagger$.

$\dagger$ This second generation Stokes line is equivalent to the 'new' Stokes lines discussed by Berk et al. (1982) and the 'vanishing' Stokes lines discussed by Aoki et al. (1998).

Article submitted to Royal Society 
Stokes smoothing for $R_{2}$

Truncating (4.25), the series for $R_{2}$, after $N$ terms we write

$$
R_{2}=\sum_{n=0}^{N-1} \epsilon^{n+1 / 2} A^{(n)} \mathrm{e}^{-i u / \epsilon}+Q
$$

The optimal truncation point is given by $N \sim|v| / \epsilon$. Substituting (4.40) into $R_{2 \tau}=$ $\epsilon^{2} R_{2 \sigma \sigma \sigma}$ gives

$$
\begin{aligned}
Q_{\tau}- & \epsilon^{2} Q_{\sigma \sigma \sigma}=\epsilon^{1 / 2} \mathrm{e}^{-i u / \epsilon}\left(\epsilon^{N+1} A_{\sigma \sigma \sigma}^{(N-1)}\right. \\
& \left.+\epsilon^{N}\left(A_{\sigma \sigma \sigma}^{(N-2)}-i\left(3 u_{\sigma} A_{\sigma \sigma}^{(N-1)}+3 u_{\sigma \sigma} A_{\sigma}^{(N-1)}+u_{\sigma \sigma \sigma} A^{(N-1)}\right)\right)\right) .
\end{aligned}
$$

Using (4.37) the right-hand side of (4.41) becomes

$$
\begin{aligned}
&-\frac{i \epsilon^{1 / 2} \mathrm{e}^{-i u / \epsilon}}{4} {\left[-\epsilon^{N+1}\left(\frac{\Gamma(N+5 / 2)\left(v_{\sigma}\right)^{3}}{v^{N+5 / 2}}+\ldots\right)+\right.} \\
&\left.\epsilon^{N}\left(-\frac{\Gamma(N+3 / 2)\left(v_{\sigma}\right)^{3}}{v^{N+3 / 2}}-3 i u_{\sigma} \frac{\Gamma(N+3 / 2)\left(v_{\sigma}\right)^{2}}{v^{N+3 / 2}}+\ldots\right)\right] .
\end{aligned}
$$

Motivated by the form of (4.42) we expect a solution of the form

$$
C(\sigma, \tau) \mathrm{e}^{(-i u-v) / \epsilon}=C(\sigma, \tau) \mathrm{e}^{-i(\sigma-\tau) / \epsilon},
$$

to be switched on across the Stokes line. Thus we set

$$
Q=C(\sigma, \tau) \mathrm{e}^{-i(\sigma-\tau) / \epsilon},
$$

to give

$$
\begin{aligned}
C_{\tau} & +3 C_{\sigma}+3 i \epsilon C_{\sigma \sigma}-\epsilon^{2} C_{\sigma \sigma \sigma} \\
& \sim-\frac{i \mathrm{e}^{i v / \epsilon} \epsilon^{N+1 / 2} \Gamma(N+3 / 2)}{4 v^{N+3 / 2}}\left(-\frac{\epsilon N\left(v_{\sigma}\right)^{3}}{v}-\left(v_{\sigma}\right)^{3}-3 i u_{\sigma}\left(v_{\sigma}\right)^{2}+\ldots\right), \\
& \sim-\frac{i \mathrm{e}^{i v / \epsilon} \epsilon^{N+1 / 2} \Gamma(N+3 / 2)}{4 v^{N+3 / 2}}\left(-\frac{\epsilon N\left(v_{\sigma}\right)^{3}}{v}+\left(v_{\sigma}\right)^{3}+v_{\tau}+3 v_{\sigma}+\ldots\right) .
\end{aligned}
$$

where we have noted that

$$
-2\left(v_{\sigma}\right)^{3}-3 i u_{\sigma}\left(v_{\sigma}\right)^{2}=v_{\tau}+3 v_{\sigma}
$$

We now change variables and write $C$ in terms of $v$ (and any other independent variable), to give, at leading-order

$$
C_{v} \sim-\frac{i \mathrm{e}^{i v / \epsilon} \epsilon^{N+1 / 2} \Gamma(N+3 / 2)}{4 v^{N+3 / 2}}\left(1+\frac{\left(v_{\sigma}^{3}\right)}{v_{\tau}+3 v_{\sigma}}\left(1-\frac{\epsilon N}{v}\right)\right) .
$$

We put $v=r \mathrm{e}^{i \theta}$, with $N=r / \epsilon+a$ and $a$ bounded as $\epsilon \rightarrow 0$. Then writing $\partial / \partial v$ in terms of $\partial / \partial \theta$ as usual gives

$$
C_{\theta} \sim \frac{\sqrt{2 \pi r}}{4 \epsilon^{1 / 2}} \exp \left[-\frac{r}{\epsilon}+\frac{r \mathrm{e}^{i \theta}}{\epsilon}-i \theta\left(\frac{r}{\epsilon}+a+\frac{1}{2}\right)\right]\left(1+\frac{\left(v_{\sigma}^{3}\right)}{v_{\tau}+3 v_{\sigma}}\left(1-\mathrm{e}^{-i \theta}\right)\right)
$$


The right-hand side is exponentially small except at the Stokes line $\theta=0$. We expand near the Stokes line by setting $\theta=\sqrt{\epsilon} \bar{\theta}$ to give

$$
C_{\bar{\theta}} \sim \frac{\sqrt{2 \pi r}}{4} \exp \left(-\frac{r \bar{\theta}^{2}}{2}\right) .
$$

Thus we have the usual error-function smoothing, and the jump in $C$ as we cross the Stokes line is $\pi / 2$. Thus across the Stokes line $R_{2}$ turns on the extra contribution

$$
-\phi_{p 1}=\frac{\pi}{2} \mathrm{e}^{-i(\sigma-\tau) / \epsilon}=\frac{\pi}{2} \mathrm{e}^{-(1+i s) / \epsilon}
$$

This contribution exactly cancels that turned on by $\phi_{1}$ so that the Stokes structure is now complete, i.e. circling the point $s=0, t=1 / \sqrt{3}$ we do indeed return to the solution we started with. We have found the leading-order solutions we found in Section 2 along with the Stokes lines we plotted in Figure 6. We note that here it was necessary for us to locate the higher-order Stokes line in order to find the leading order approximations whereas in deriving these approximations from the exact solution we did not.

Thus we have indeed been able in this case to find the Stokes structure directly from the equation, without using the integral formulation of the solution.

\section{Direct application of 'factorial over power' ansatz}

Although $\phi^{(n)}$ can be obtained exactly in the present case for all $n$, this is unlikely to be possible in general, even for linear equations. Since to identify the Stokes structure all we need is the asymptotic behaviour of $\phi^{(n)}$ for large $n$, it is therefore natural to ask whether this can be obtained directly, that is, whether we can approximate the equation for $\phi^{(n)}$ for large $n$ and then solve, rather than solving exactly and then approximating. In this section, we examine the possibilities and difficulties of obtaining the late terms through a direct application of a factorial/power ansatz, as is possible for ordinary differential equations (see Chapman et al. (1998)). Thus we suppose that

$$
\phi^{(n)} \sim A \frac{\Gamma(2 n+\gamma)(-1)^{n}}{u^{2 n+\gamma}}
$$

as $n \rightarrow \infty$ where $A, u$ and $\gamma$ may be functions of $\sigma$ and $\tau$, but are independent of $n$. Substituting (5.1) into (3.9) and equating coefficients at $\mathcal{O}\left(n \phi^{(n)}\right), \mathcal{O}\left(\log (n) \phi^{(n)}\right)$, and $\mathcal{O}\left(\phi^{(n)}\right)$ we have

$$
\begin{aligned}
u_{\tau}+\left(u_{\sigma}\right)^{3} & =0 \\
\gamma_{\tau}+3 \gamma_{\sigma}\left(u_{\sigma}\right)^{2} & =0 \\
A_{\tau}+3\left(u_{\sigma}\right)^{2} A_{\sigma}+3 u_{\sigma} u_{\sigma \sigma} A & =0 .
\end{aligned}
$$

Writing $p \equiv u_{\tau}, q \equiv u_{\sigma}$, and solving (5.2) by Charpit's method (see e.g.Ockendon et al. (1999)) gives

$$
\tau=T+\tau_{0}(S), \quad \sigma=3 q_{0}^{2} T+\sigma_{0}(S), \quad u=2 q_{0}^{3} T+u_{0}(S),
$$


where the initial data $u=u_{0}(S)$ is given on the curve $\tau=\tau_{0}(S), \sigma=\sigma_{0}(S)$, and the values of $p_{0}(S)$ and $q_{0}(S)$ are determined from

$$
p_{0}+q_{0}^{3}=0, \quad \frac{\mathrm{d} u_{0}}{\mathrm{~d} S}=p_{0} \frac{\mathrm{d} \tau_{0}}{\mathrm{~d} S}+q_{0} \frac{\mathrm{d} \sigma_{0}}{\mathrm{~d} S} .
$$

The factorial/power divergence is generated by repeatedly differentiating the singularities of the early terms $\left(\phi^{(0)}\right.$ and $\left.\phi^{(1)}\right)$. Thus the boundary condition we must impose is that $u=0$ at such a singularity. There are two singularities to consider, the static singularity at $s=i,(\sigma=\tau)$, and the moving singularity at $s=i-t$, $(\sigma=0)$.

\section{(a) Static singularity}

To determine the late terms generated by the static singularity we must impose

$$
u_{0}=0, \quad \sigma_{0}=S, \quad \tau_{0}=S .
$$

Then (5.6) gives $q_{0}\left(q_{0}^{2}-1\right)=0$. The zero solution gives only the trivial solution $u=0$, while the others give $u= \pm(\sigma-\tau)$. Since changing the sign of $u$ simply multiplies the function $A$ by a constant, without loss of generality we can take

$$
u=\sigma-\tau \text {. }
$$

Equation (5.3) for $\gamma$ and (5.4) for $A$ now become

$$
\begin{aligned}
\gamma_{\tau}+3 \gamma_{\sigma} & =0 \\
A_{\tau}+3 A_{\sigma} & =0 .
\end{aligned}
$$

Hence $\gamma=\gamma(\sigma-3 \tau)$ and $A=A(\sigma-3 \tau)$. Since $\phi_{1}$ has a double pole at $\sigma=\tau$ we must have $\gamma(-2 \tau)=0$, i.e. $\gamma \equiv 0$. To determine $A$ we need to match with an inner region in the vicinity of the singularity $\sigma-\tau=0$. Changing variables by writing

$$
\begin{aligned}
\sigma-\tau & =\epsilon z, \\
\sigma-3 \tau & =y, \\
\phi & =-\frac{i}{2} \log \epsilon-\frac{i}{2} \psi,
\end{aligned}
$$

to leading-order equation (1.10) becomes

$$
\psi_{z z z}+\psi_{z}-\frac{1}{z}=0
$$

To match with the outer solution we expand $\psi$ as $z \rightarrow \infty$ as

$$
\psi=\log z+\sum_{n=1}^{\infty} \frac{a_{n}(y)}{z^{2 n}},
$$

where the $a_{n}$ satisfy

$$
\begin{aligned}
a_{n}+(2 n-2)(2 n-1) a_{n-1} & =0 \\
a_{1} & =1 .
\end{aligned}
$$


Hence

$$
a_{n}=(-1)^{n+1} \Gamma(2 n)
$$

This is the outer limit of the one-term inner expansion. To match we calculate the one-term inner limit of the outer expansion (5.1). In the inner coordinates

$$
\epsilon^{2 n} \phi^{(n)} \sim A(y) \frac{\Gamma(2 n)(-1)^{n}}{z^{2 n}} .
$$

Matching now gives $A=i / 2$, so that

$$
\phi^{(n)} \sim \frac{i(-1)^{n} \Gamma(2 n)}{2(\sigma-\tau)^{2 n}} .
$$

Thus we have been able to obtain the later terms $\phi_{1}^{(n)}$ without any difficulty.

\section{(b) Moving singularity}

To determine the late terms generated by the moving singularity we must impose

$$
u_{0}=0, \quad \sigma_{0}=0, \quad \tau_{0}=S .
$$

Unfortunately (5.6) then gives $p_{0}=q_{0}=0$, and the rays never make it away from the boundary. This occurs because the boundary curve is a characteristic of the equation.

The solution we require must be in the form of a fan of rays, all emanating from the initiation of the singularity at $\sigma=0, \tau=0$. To find such a solution we must drop the second boundary condition (5.6) and impose instead that all the rays go through $\sigma=0, \tau=0$. Thus in (5.5) we have $u_{0}=\sigma_{0}=\tau_{0}=0$, but $q_{0}$ is arbitrary. Eliminating $q_{0}$ gives

$$
u=\left(\frac{4 \sigma^{3}}{27 \tau}\right)^{1 / 2}
$$

where, as before, we have taken the plus sign without loss of generality. The equation for $\gamma$ then becomes

$$
\gamma_{\tau}+\frac{\sigma}{\tau} \gamma_{\sigma}=0
$$

and the equation for $A$ is

$$
A_{\tau}+\frac{\sigma}{\tau} A_{\sigma}+\frac{1}{2 \tau} A=0
$$

Hence $\gamma=\gamma(\sigma / \tau)$ and $A=\tau^{-1 / 2} B(\sigma / \tau)$ and we have

$$
\phi^{(n)} \sim B\left(\frac{\sigma}{\tau}\right)(-1)^{n} \frac{\Gamma(2 n+\gamma(\sigma / \tau))}{\sqrt{\tau}}\left(\frac{27 \tau}{4 \sigma^{3}}\right)^{n+\frac{\gamma(\sigma / \tau)}{2}} .
$$

As $\sigma \rightarrow 0, \phi^{(1)} \sim i / 2 \sigma^{2}$. For this to agree with (5.23) requires $\gamma(0)=-1 / 2$, and $B(y) \sim y^{1 / 4}$ as $y \rightarrow 0$. To determine the functions $\gamma$ and $B$ we need to match with an inner region in the vicinity of the singularity as before. 
We introduce the inner coordinates

$$
\begin{aligned}
y & =\frac{\sigma}{\tau} \\
\epsilon z & =\left(\frac{4 \sigma^{3}}{27 \tau}\right)^{1 / 2}=u, \\
\phi & =-\frac{i}{2} \log \epsilon+\frac{i \psi}{2} .
\end{aligned}
$$

The leading-order inner problem is

$$
\begin{aligned}
2 y \psi_{y}+\frac{\psi_{z}}{9 z}\left(9 z^{2}-1\right) & +\frac{2 y \psi_{y z}}{3 z}+\psi_{z z}+\frac{8 y^{3} \psi_{y y y}}{27 z^{2}} \\
+ & \frac{4 y^{2} \psi_{z y y}}{3 z}+2 y \psi_{z z y}+z \psi_{z z z}+\frac{i}{y-1}=0 .
\end{aligned}
$$

Expanding for large $z$ gives

$$
\psi=-\log \left(\frac{3^{3 / 2} z(y-1)}{2 y^{3 / 2}}\right)+\sum_{n=1}^{\infty} \frac{B_{n}(y)}{z^{2 n}},
$$

where

$$
\begin{aligned}
-y B_{n}^{\prime}+n B_{n}= & -\frac{4(3 n-1)(3 n-2)(n-1)}{9} B_{n-1}+\frac{4(n-1)(3 n-2)}{3} y B_{n-1}^{\prime} \\
& -\frac{4(n-1)}{3} y^{2} B_{n-1}^{\prime \prime}+\frac{4}{27} y^{3} B_{n-1}^{\prime \prime \prime}, \quad n \geq 2 \\
-y B_{1}^{\prime}+B_{1}= & -\frac{8 y^{3}}{27(y-1)^{3}} .
\end{aligned}
$$

The boundary conditions on these equations come from the initial condition on the equation, since $\tau=0$ is contained in the inner region. Since the initial condition is taken care of by the logarithm in (5.24), the remaining terms must be zero on the boundary $\tau=0$. This translates to the condition that

$$
B_{n}=o\left(y^{n}\right) \quad \text { as } y \rightarrow \infty .
$$

The late terms will be of the form

$$
B_{n}(y) \sim(-1)^{n} \Gamma(2 n-1 / 2) b(y),
$$

but unfortunately $b(y)$ cannot be determined by applying this late terms ansatz to (5.25) (on applying the ansatz and equating coefficients of powers of $n, b(y)$ remains arbitrary).

The solution for $B_{n}$ may be written as

$$
B_{n}(y)=\frac{(-1)^{n} \Gamma(2 n) 4^{n} y^{3 n}}{27^{n}(y-1)^{2 n}}+\frac{(-1)^{n+1} \Gamma(2 n) 4^{n}}{27^{n}} \sum_{j=0}^{n-1} a_{n, j} y^{n-j}
$$

where

$$
j(2 n-2)(2 n-1) a_{n, j}=(2 n+j-1)(2 n+j-2)(2 n+j-3) a_{n-1, j-1},
$$


and, to satisfy the initial condition,

$$
a_{n, 0}=1
$$

Thus

$$
a_{n, j}=\frac{\Gamma(2 n+j)}{\Gamma(2 n) \Gamma(j+1)}
$$

Matching now requires a steepest descents analysis of (5.28), exactly as in Section b. Of course, we have simply reconstructed the solution of Section b in a different set of coordinates. The reason for this is obvious when we note that in the inner region, where $y$ and $z$ are $\mathcal{O}(1), \sigma$ and $\tau$ are both $\mathcal{O}(\epsilon)$. Writing $\sigma=\epsilon \bar{\sigma}, \tau=\epsilon \bar{\tau}$ the inner equation is

$$
\phi_{\bar{\tau}}=\phi_{\bar{\sigma} \bar{\sigma} \bar{\sigma}}+\frac{i}{2(\bar{\sigma}-\bar{\tau})}
$$

Thus in fact we retrieve the full problem, with $\epsilon$ simply scaled out of the equation. This happens because of the particularly simple equation we are considering, and will not be true in general.

Both the static singularity, $\sigma=\tau$, and the moving singularity, $\sigma=0$, are in the inner region. This is associated with the fact that $u$ is given by a fan of rays emanating from the origin. The situation is analogous to a diffraction problem in which a ray in geometrical optics strikes a point of nonanalyticity of a scatterer. A fan of diffracted rays is generated, and the amplitude along each ray can only be determined by solving an inner diffraction problem. The inner problem is the diffraction problem to determine our ray amplitude function $B$. Note though that by matching (5.23) and (5.24) we can determine that $\gamma$ must be constant and therefore $\gamma \equiv-1 / 2$.

\section{(c) Second-generation singularity}

Let us now try and apply the factorial/power ansatz to equation (4.27) to determine the late terms of the remainder $R_{2}$.

Since we expect the series to diverge in the form of factorial/power, following Chapman et al. (1998) we propose the ansatz

$$
A^{(n)} \sim \frac{B(\sigma, \tau) \Gamma(n+\gamma)}{v(\sigma, \tau)^{n+\gamma}}
$$

as $n \rightarrow \infty$, where $v, B$ and $\gamma$ may be functions of $\sigma$ and $\tau$, but are independent of $n$. Substituting (5.32) into (4.27) we find that at leading-order in $n$

$$
v_{\tau}+\frac{\sigma}{\tau} v_{\sigma}=\left(v_{\sigma}\right)^{3}+i \sqrt{\frac{3 \sigma}{\tau}}\left(v_{\sigma}\right)^{2}
$$

The divergence of the series is due to the singularity of $A^{(0)}$ at $\sigma=3 \tau$, what we shall refer to as the second-generation singularity. We note that this is a singularity in the late terms only and not in the terms $\phi^{(n)}$ for $n=\mathcal{O}(1)$. 
The boundary condition on (5.33) is therefore $v=0$ on $\sigma=3 \tau$. In principle we can solve (5.33) by Charpit's method. However, a scaling analysis shows that (5.33) has solutions of the form

$$
v=\tau f(\eta) \quad \text { where } \eta=\frac{\sigma}{\tau},
$$

and since the boundary condition is applied at $\eta=3$ our solution will be of this form. We find that $f$ satisfies

$$
\begin{aligned}
f & =\left(f^{\prime}\right)^{3}+i \sqrt{3 \eta}\left(f^{\prime}\right)^{2}, \\
f(3) & =0,
\end{aligned}
$$

with solution

$$
f=i\left(\eta-1-\left(\frac{4 \eta^{3}}{27}\right)^{1 / 2}\right)
$$

giving

$$
v=i\left(-\sqrt{\frac{4 \sigma^{3}}{27 \tau}}+\sigma-\tau\right)
$$

This is just the difference of the two singulants, (5.8) and (5.20), as we know it must be in order for our Stokes structure to be consistent. At the next order in $n$ we find

$$
\gamma_{\tau}+3 \gamma_{\sigma}=0
$$

so $\gamma=\gamma(\sigma-3 \tau)$. At the next order in $n$, after much cancellation, the equation for $B$ is

$$
B_{\tau}+3 B_{\sigma}=0
$$

Thus $B=B(\sigma-3 \tau)$, and

$$
A^{(n)} \sim \frac{\Gamma(n+\gamma) B(\sigma-3 \tau)}{v^{n+\gamma}} .
$$

Now on the face of it we cannot determine $B$, even from matching with an inner region in the vicinity of the singularity. This is because the singularity is at $\sigma=3 \tau$, so that we can only determine $B(0)$ from such an analysis. However, from (4.28) we find that

$$
\Gamma(n+\gamma(z)) B(z) \sim f_{n}(\eta) f(\eta)^{n+\gamma(z)}\left(\frac{z}{\eta-3}\right)^{\gamma(z)-1 / 2},
$$

where $z=\sigma-3 \tau$. Even without knowing $f_{n}(\eta)$, we see that the only possibility is $\gamma=$ constant and

$$
B(z)=\Lambda z^{\gamma-1 / 2},
$$


where $\Lambda=$ constant. Now,

$$
v \sim-i(\sigma-3 \tau)^{2} / 12 \tau, \quad \text { as } \sigma \rightarrow 3 \tau,
$$

so for (5.37) to be consistent with (4.26) requires that $\gamma=1 / 2$. Thus $B$ must in fact also be constant.

Motivated by (5.39) we change variables from $\sigma$ to $y$ in the inner region by writing

$$
\sigma=3 \tau+\epsilon^{1 / 2} \tau^{1 / 2} y,
$$

so that with $R_{2}=A \mathrm{e}^{-i u / \epsilon}$, at leading-order in $\epsilon$, the equation $R_{2 \tau}=\epsilon^{2} R_{2 \sigma \sigma \sigma}$ becomes

$$
2 \tau A_{\tau}+y A_{y}+A=-6 i A_{y y},
$$

with the first term (4.26) implying that

$$
A \sim \frac{\sqrt{3 \pi} \mathrm{e}^{-i \pi / 4}}{2 y} \quad \text { as } y \rightarrow \infty .
$$

Expanding for large $y$ as

$$
A \sim \sum_{n=0}^{\infty} \frac{c_{n}(\tau)}{y^{2 n+1}}
$$

we have

$$
\begin{aligned}
& c_{n}=12 i(n-1 / 2) c_{n-1}, \\
& c_{0}=\frac{\sqrt{3 \pi} \mathrm{e}^{-i \pi / 4}}{2} .
\end{aligned}
$$

Hence

$$
c_{n}=\frac{\sqrt{3} \mathrm{e}^{-i \pi / 4}(12 i)^{n} \Gamma(n+1 / 2)}{2} .
$$

In the inner coordinates, from (5.37) and (5.39),

$$
\epsilon^{n+1 / 2} A^{(n)} \sim \frac{B \Gamma(n+1 / 2)(12 i)^{n+1 / 2}}{y^{2 n+1}} .
$$

Matching with (5.40) gives

$$
B=\frac{\sqrt{3} \mathrm{e}^{-i \pi / 4}}{2(12 i)^{1 / 2}}=-\frac{i}{4},
$$

so that

$$
A^{(n)}=-\frac{i \Gamma(n+1 / 2)}{4 v^{n+1 / 2}},
$$

as required.

Article submitted to Royal Society 


\section{Conclusions}

We have begun to develop a methodology for calculating exponentially small terms beyond all orders in the asymptotic expansions of the solutions to partial differential equations. We used an example motivated by the geometric model for crystal growth to demonstrate this methodology. The example was chosen to be linear so that the exact solution could be formulated in terms of an integral using a Fourier transform. In Section 2 a steepest descent analysis of this integral was performed to identify various saddle point, end point and pole contributions to the approximation; and the Stokes lines across which they are turned on or off. The existence of such a representation of the solution provides a good check on the more direct methods of Sections 3-5.

In Section 3 we found the full naïve asymptotic expansion of the solution directly from the equation. The Stokes lines and subdominant exponentials may be identified by optimally truncating this expansion and smoothing the Stokes lines, as shown by Berry (1989) and by Chapman et al. (1998). However, in this case we found that the $n^{\text {th }}$ term in the expansion was a sum of terms of the form facto$\mathrm{rial} /$ power, which needed to be approximated as $n \rightarrow \infty$. Using a Watson transform to recast the sum as an integral and performing a steepest descents analysis, we found that the approximation exhibited Stokes phenomenon, in that contributions to the approximation of the late terms may be switched on or off across what we termed higher-order Stokes lines. Since the late terms in the series are responsible for Stokes lines, such a higher-order Stokes line switches on and off Stokes lines themselves.

In Section 4 we performed the Stokes smoothing and identified the Stokes lines and the exponentials they switch on. However, the picture was still not complete. We found that one of the exponentials switched on across a Stokes line was in fact given by a divergent asymptotic expansion, which itself exhibited Stokes phenomenon. We have called such a Stokes line a second-generation Stokes line. This second generation Stokes line is equivalent to the 'new' Stokes lines discussed by Berk et al. (1982) and the 'vanishing' Stokes lines discussed by Aoki et al. (1998).

Finally, in Section 5, we considered the possibility of identifying the late terms directly through a factorial/power ansatz as $n \rightarrow \infty$ in the same way as Chapman et al. (1998), since although in the example we considered we are able to find the full asymptotic series of the solution exactly, this is unlikely to be the case in general. The factorial/power ansatz leads to an eikonal equation for the "phase" and a transport equation for the premultiplying amplitude, with boundary conditions given by the position of the singularities in the leading-order problem. For some singularities these equations were easy to solve, but for some the boundary data turned out to be given along a characteristic of the eikonal. Such singularities produce eikonal solutions which are a fan of rays. This situation is analogous to the Geometrical Theory of Diffraction (see Keller $(1958,1962)$ ), in which a ray striking a point of nonanalyticity in a boundary generates a cone of diffracted rays. The amplitude along these rays can only be determined by solving a canonical inner diffraction problem. Exactly the same happens in the present situation; we have a canonical inner problem to solve for our premultiplying amplitude.

The authors would like to acknowledge the referees for their very helpful and thorough reports, and in particular for the suggestion to include a discussion of the Borel plane. 
They would also like to acknowledge many helpful discussions with Prof. J.R. King, Dr C. J. Howls, Dr A. Olde-Daalhuis and Dr R. Tew. SJC would like to acknowledge the support of EPSRC through grant GR/R18642/01. DBM is in receipt of an EPSRC studentship.

\section{References}

Amick, C. J. \& MCLEOD, J. B. 1990 A singular pertubation problem in needle crystals. Arch. Rat. Mech. Anl. 109, 139-171.

Aoki, T., Kolke, T. \& TAkeI, Y. 1998 Vanishing of stokes Stokes curves. In Microlocal analysis and complex fourier analysis (ed. K. F. T. Kawai), pp. 165176. Kyoto University Press.

Berk, H. L., Nevis, W. M. \& Roberts, K. V. 1982 New Stokes lines in WKB theory. J. Math. Phys. 23(6), 988-1002.

Berry, M. V. 1989 Uniform asymptotic smoothing of Stokes discontinuities. Proc. Roy. Soc. London A422, 7-21.

Brower, R. C., Kessler, D. A., Koplik, J. \& Levine, H. 1983 Geometrical approach to moving-interface dynamics. Phys. Rev. Lett. 51, 1111-1114.

Chapman, S. J., King, J. R. \& Adams, K. L. 1998 Exponential asymptotics and Stokes lines in nonlinear ordinary differential equations. Proc. Roy. Soc. London A454, 2733-2755.

Danlhuis, A. B. O., Chapman, S. J., King, J. R., Ockendon, J. R. \& Tew, R. H. 1995 Stokes phenomenon and matched asymptotic expansions. SIAM J. Appl. Math 55(6), 1469-1483.

Dingle, R. B. 1973 Asymptotic Expansions: their Derivation and Interpretation. Academic Press.

Howls, C. J., Langman, P. J. \& Danlhuis, A. B. O. 2005 On the higher order Stokes phenomenon. Preprint 2003.

Keller, J. B. 1958 A geometrical theory of diffraction. In Calculus of Variations and Its Applications, Proceedings of Symposia in Applied Mathematics (ed. L. M. Graves), , vol. 8, pp. 27-52. McGraw-Hill, New York, math. Revs. 20, 103, 1959.

Keller, J. B. 1962 The geometrical theory of diffraction. J. Opt. Soc. Am 52, $116-130$

KING, J. R. 1998 Interacting Stokes lines. In Toward the Exact WKB Analysis of Differential Equations, Linear or Nonlinear (ed. C. Howls, T. Kawai \& Y. Takei), pp. 165-176. Kyoto University Press.

Kruskal, M. D. \& Segur, H. 1991 Asymptotics beyond all orders in a model of chrystal growth. Stud. Appl. Math. 85, 129-181.

Ockendon, J. R., Howison, S., Lacey, J. \& Movchan, A. 1999 Applied Partial Differential Equations. Oxford University Press. 